\title{
An Experimental Investigation of Blocking by Partial Barriers in a Rotating Baroclinic Annulus
}

\author{
S. D. Marshall \& P. L. Read \\ [Atmospheric, Oceanic \& Planetary Physics, Clarendon Laboratory, Parks Road, Oxford, OX1 3PU]
}

\begin{abstract}
We present a series of experimental investigations in which a differentially-heated annulus was used to investigate the effects of topography on rotating, stratified flows with similarities to the Earth's atmospheric or oceanic circulation. In particular, we compare and investigate blocking effects via partial mechanical barriers to previous experiments by the authors utilising azimuthally-periodic topography.

The mechanical obstacle used was an isolated ridge, forming a partial barrier, employed to study the difference between partially blocked and fully unblocked flow. The topography was found to lead to the formation of bottom-trapped waves, as well as impacting the circulation at a level much higher than the top of the ridge. This produced a unique flow structure when the drifting flow and the topography interacted in the form of an 'interference' regime at low Taylor number, but forming an erratic 'irregular' regime at higher Taylor number. The results also showed evidence of resonant wavetriads, similar to those noted with periodic wavenumber-3 topography by Marshall and Read (2015), though the component wavenumbers of the wave-triads and their impact on the flow were found to depend on the topography in question. With periodic topography, wave-triads were found to occur between both the baroclinic and barotropic components of the zonal wavenumber- 3 mode and the wavenumber-6 baroclinic component, whereas with the partial barrier two nonlinear resonant wavetriads were noted, each sharing a common wavenumber-1 mode.
\end{abstract}

\section{Keywords}

Blocking, Topography, Annulus, Wave-Triads, Trapped Waves. 


\section{Introduction}

Surface topography is well known to exert a major influence on the atmospheric circulation, determining the existence and location of stationary topographic waves superposed on the zonal flow. Another notable effect may be the possible formation of distinct, metastable, coexisting circulation regimes, as explained by Charney and DeVore (1979). In simple, spectrally truncated models, topographical forcing was shown to lead to the development of either a 'low zonal index' flow or a 'high zonal index' flow as alternative states, depending upon initial conditions. However, the existence of these states in fully continuous flows is still in question, as noted by Tung and Rosenthal (1985) and Cehelsky and Tung (1987). The former state (also known as "blocked flow") was defined by Charney and DeVore as having “a strong wave component and a weaker zonal [flow] component locked close to linear resonance”. This locking may be associated with nonlinear interactions of the topography with the zonal flow. The latter state (also known as a "zonal flow state”) has "a weak wave component and a stronger zonal component much further from linear resonance”. Both of these states may be stable at the same location in parameter space (sometimes also referred to as metastable or quasi-stable), giving rise to the existence of multiple equilibria or other forms of multi-stability. Transitions between the two states may be forced by perturbations due to baroclinic instabilities of the topographic waves, for example. Egger (1978) described blocking as arising from the nonlinear interactions of slowly drifting free waves and stationary waves caused by "geographically fixed" forcing, such as due to topographic ridges.

In order to investigate blocking and related topographic impacts, a differentially-heated annulus experiment with topography (as discussed e.g. by Hide and Mason 1975, Weeks et al. 1997, and Read and Risch 2011) allows for controllable baroclinic flows in a laboratory environment, and forms the framework of the experiments conducted. The experimental arrangement is similar in design to that of Marshall and Read (2015), which utilised a different design of topographic base to investigate topographic resonance. 


\subsection{Blocking in Geophysical Flows}

Some of the earliest references to the importance of blocking to the atmospheric circulation had been noted by Berggren et al. (1949), who detailed the synoptic-scale disturbances that affect both the local weather and the climate. An increased persistence and frequency of blocked states, linked to both increasing levels of greenhouse gases and associated climate change, has been suggested by Woollings (2010) to be an explanation for recent severe weather events, especially in Europe. In a related way, Weeks et al. (1997) noted that blocking events (“persistent blocking anomalies”) typically occur over a timescale of 10-100 days, which happens to be the same as that of more general atmospheric intraseasonal low frequency variability (LFV). One manifestation of LFV is therefore likely to be associated with slow, cyclic oscillations or vacillations between mobile zonal states and near-stationary blocked states in the atmosphere. The extent to which this interaction occurs, the conditions for it to do so, and the mechanisms and factors affecting the timescales involved are amongst the main motivations for the present study of blocking phenomena in rotating annulus experiments.

One question about blocking found in the literature concerns the issue of the existence of multiple equilibria or other intransitive states. Most notably, Charney and DeVore (1979), Charney and Straus (1980) and Reinhold and Pierrehumbert (1982) suggested the idea that both the blocked and zonal regimes could be stable at the same parametric location, as alternative states that the system could 'choose' to exhibit, depending upon the initial conditions. A change in the parameters might then lead to bifurcations to time-dependent regimes in which the flow vacillates (periodically or chaotically) between these two states, and that these transitions are analogous to many of the atmospheric anomalies that are observed. On the other hand, Tung and Rosenthal (1985) and Cehelsky and Tung (1987) claimed that, although multiple equilibria are physically possible in some systems, they cannot exist in the real atmosphere. They suggested that previous results demonstrating multiple equilibria were caused by unrealistic topography or overly-truncated representations of nonlinear interactions. 
In contrast, Risch (1999) and Read and Risch (2011) claimed to find evidence in the laboratory for multiple equilibria and intransitivity in a thermally-forced, rotating annulus, both with and without topography. While the existence of multiple equilibria in the classical case without topography was, by this point, no longer in question even for higher-order models, the impact of topography on the occurrence of multiple stable flow states was still under debate. The topography used in both Risch's and Risch and Read's experiments had a simple wavenumber-3 shape, suggesting that the low-order models that found multiple equilibria with similar topography were not merely seeing a false positive due to their 'overly-truncated nonlinear interactions', as alleged by Tung and Rosenthal (1985) and Cehelsky and Tung (1987). By extension, Risch (1999) also noted that this would imply that multiple equilibria should also be possible in a baroclinic planetary atmosphere with topography. Risch does admit that the results taken were merely qualitative in nature and only involve simple topography, however, and so suggests that similar experiments should be carried out with more quantitative analyses. Supporting the other side of the argument, Tian et al. (2001) compared similar numerical and laboratory studies on a rotating, barotropic annulus, finding stable multiple equilibria to be prevalent in the model simulations, but not to exist at all in the laboratory. The physical annulus experiment still produced both zonal and blocked regimes, but they were found to be meta-stable, occurring sporadically but with sudden and irregular transitions between them. However, it was possible that the lack of genuine and robust multiple equilibria in the experiments could be due to the fact that the flow system in question was barotropic.

In an annulus experiment, although the direct geometric and dynamical similarity with the Earth's atmosphere is limited and imperfect, the nonlinear interactions will not be unphysically truncated. This gives access to a range of perfectly 'realistic' flow states in a number of important respects, if boundary effects with the inner and outer walls (and other boundary conditions dissimilar to the real atmosphere) can be judged to be relatively insignificant to the system as a whole. In this way, the sensitivity of the problem under investigation to truncation effects can be studied, complementing the results from finite resolution numerical models, regardless of complexity. As such, it appears that a suitable approach would be both to employ a baroclinic annulus flow, thus testing the sensitivity of Tian 
et al.'s (2001) barotropic results to the presence of additional vertical degrees of freedom, and to use quantitative analyses, systematically exploring parameter space and thus verifying and extending the qualitative findings of Risch (1999), Read and Risch (2011) and Risch and Read (2015). Combining this with the fully nonlinear interactions of a physical annulus, an investigation into the existence of multiple equilibria could be launched, stringently putting to the test the assertions of Tung and Rosenthal (1985) and Cehelsky and Tung (1987).

There are many ways to study blocking, especially when using numerical models, but within a baroclinic annulus in the laboratory, one of the best methods of investigation, in terms of both simplicity and versatility, is via the use of partial barriers that obstruct just a fraction of the annular channel. Partial barriers may be used to block part of the flow (either radially or vertically), and allow a comparison between blocked and unblocked flow. The physical structure of a partial barrier most resembles that of the margins of a continental shelf on the ocean basin - thus also permitting a comparison with the effects of topography on aspects of oceanic circulation. In previous annulus experiments with a full vertical barrier, Wordsworth (2009) encountered a quasi-barotropic jet, despite the obstruction to the circulation, which gained in strength as it flowed towards the upstream side of the barrier, and ended with a large, persistent, azimuthally-trapped gyre. The author noted that this trapped flow structure was equivalent to the western boundary currents of oceanic flows, similar to the observations of Rayer et al. (1998). These trapped waves and boundary currents are amongst the most prominent effects of the flow around continental shelves, as discussed and reviewed e.g. by Huthnance (1989). Hence, for purposes of comparison, the present partial barriers study allows an examination of the relative importance of the trapped wave to the general circulation in a flow that is only partially blocked.

Furthermore, as mentioned previously, there are many similarities in experimental design and parameter space under investigation between the present Partial Barrier experiments and those of Marshall and Read (2015). Marshall and Read employed a zonally periodic wavenumber-3 topographic base, and noted evidence of topographic resonance and associated bifurcations, leading to oscillations. These resonant oscillations were associated with the occurrence of near-resonant wave-triads between topographically-forced, stationary and drifting waves in a region of dominant stationary wavenumber- 
3 flow structure. In addition, unlike Risch (1999) and Read and Risch (2011), Marshall and Read (2015) found no evidence of multiple equilibria. This being the case, the particular goals of the present work were: (a) to explore whether strong topographically resonant bifurcations and oscillations and resonant wave triads can be observed in the presence of isolated topography, and (b) to investigate the impact of isolated topography on the occurrence of multiple baroclinic flow states, in comparison with earlier work on baroclinic wave flows with and without periodic bottom topography.

\subsection{Outline}

Section 2 presents a detailed account of the experimental apparatus that this project utilised, as well as the methodology that was employed, and defines some of the key dimensionless parameters needed to describe the most relevant parameter space. Section 3 presents an overview of the results of the conducted experiments, with a more detailed analysis of the wave interactions in Section 4 and the vertical structure in Section 5. This is followed by a discussion and summary of conclusions in Section 6, which examines how successful the investigation has been at achieving the aims and objectives set out in the previous section and discusses the physical implications of the results gathered.

\section{Experimental Arrangement}

\subsection{Non-Dimensional Numbers}

The principal flow regimes in typical rotating annulus experiments are generally characterised mainly by two dimensionless numbers defining a tractable parameter space. The Taylor number for an annulus experiment, as described in Fowlis and Hide (1965), is defined as:

$$
\mathcal{T}=\frac{4 \Omega^{2}(b-a)^{5}}{v^{2} d}
$$


where $v$ is the kinematic viscosity, $a$ is the inner radius, $b$ is the outer radius, $d$ is the height of the annulus and $\Omega$ is the rate of rotation. The Taylor number typically represents the squared ratio of the Coriolis forces (the numerator) to the viscous forces (the denominator) acting upon a fluid, such that a large value implies a less strongly damped flow.

Secondly, the Hide number (sometimes also known as the Thermal Rossby number, such as in Hide (1958) and Risch (1999), amongst others) for an annulus experiment is defined as:

$$
\Theta_{\mathrm{T}}=\frac{\alpha g d \Delta T}{\Omega^{2}(b-a)^{2}}
$$

where $\alpha$ is the volumetric thermal expansion coefficient, $g$ is the gravitational acceleration and $\Delta T$ is the imposed temperature difference. A full derivation can be found in Holton (1992), for example. Roughly, the Hide number gives the ratio of inertial forces (the numerator) to the Coriolis forces (the denominator) typically acting upon a fluid if the velocity scale is taken to be the geostrophic thermal wind shear.

For a rotating annulus experiment (or similar), the rotation rate and the temperature difference are the main sources of control. Hence, together with the Prandtl number (kept constant in the present investigation), these dimensionless numbers can be taken to adequately define the parameter space within which the experiments take place, as in Hide and Mason (1975), Weeks et al. (1997), and Read and Risch (2011), amongst others.

\subsection{Equipment Description}

Accounts of the experimental arrangement in question can be found more fully in Wordsworth et al. (2008) and Marshall and Read (2015). Its dimensions, as well as several other relevant experimental parameters, are given in Table 1. 
Table 1: Important experimental parameters

\begin{tabular}{|l|l|l|}
\hline Radius of inner cylinder & a & $4.5 \pm 0.05 \mathrm{~cm}$ \\
\hline Radius of outer cylinder & b & $14.3 \pm 0.05 \mathrm{~cm}$ \\
\hline Average depth of annulus (mid-radius lid to base minimum) & d & $24.5 \pm 0.05 \mathrm{~cm}$ \\
\hline Density of water-glycerol mixture at $20^{\circ} \mathrm{C}$ & $\rho$ & $1.044 \pm 5 \times 10^{-4} \mathrm{kgm}^{-3}$ \\
\hline Kinematic viscosity of water-glycerol mixture at $20^{\circ} \mathrm{C}$ & $v$ & $1.58 \times 10^{-6} \pm 3 \times 10^{-8} \mathrm{~m}^{2} \mathrm{~s}^{-1}$ \\
\hline Thermal expansion coefficient of water-glycerol mixture at $20^{\circ} \mathrm{C}$ & $\alpha$ & $3.16 \times 10^{-4} \pm 1 \times 10^{-6} \mathrm{~K}^{-1}$ \\
\hline
\end{tabular}

The working fluid was a mixture of water and glycerol of density $1.044 \pm 5 \times 10^{-4} \mathrm{kgm}^{-3}$, and of kinematic viscosity $1.58 \pm 0.03 \times 10^{-6} \mathrm{~m}^{2} \mathrm{~s}^{-1}$. This allowed a suspension of $350-500 \mu \mathrm{m}$ diameter pliolite tracer particles to be neutrally buoyant. For visualisation, an array of strips of 120 Maxilux Prostrip white light LEDs, arranged over five layers (120 LEDs per layer) surrounded the annulus, generating light that was allowed to pass through transparent slits to form flat, collimated light sheets at those layers. This is illustrated in Figure 1. A camera was mounted above the annulus on a tripodshaped superstructure, with a conical baffle blocking out all external light between it and the acrylic lid. With this arrangement the camera could see the motion of the tracer particles, and thus the flow structure, at any one of the five levels indicated in Figure 1. By switching quickly between the levels the vertical structure could also be resolved. 


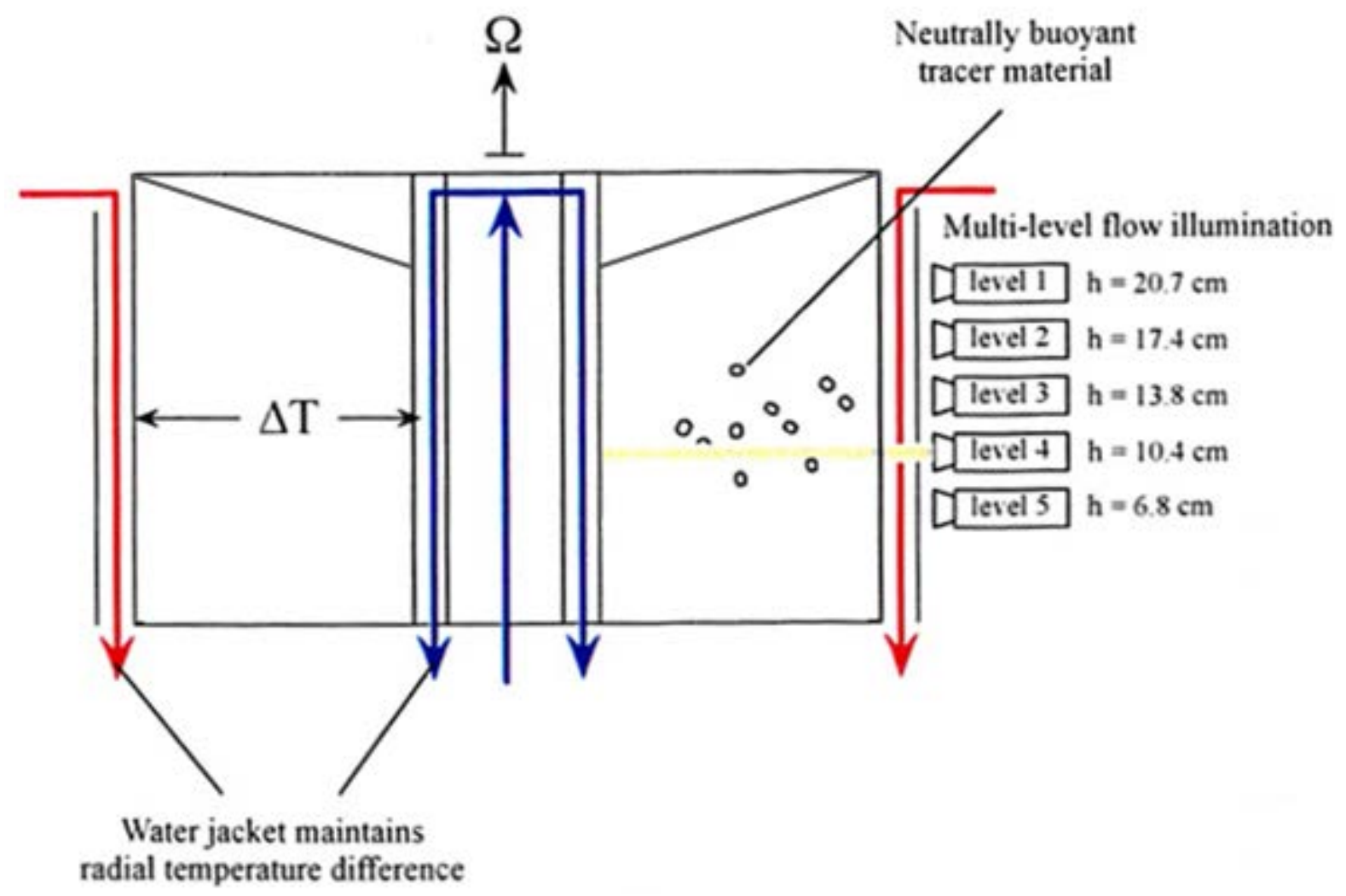

Figure 1: Schematic of the lighting array, sloped lid and heating system in side view [adapted from Wordsworth et al. (2008)].

\subsubsection{Data Acquisition}

A Firewire (type: DFK 31BF03) camera, chosen for its high image quality and lossless compression, was attached to a Lenovo ThinkPad SL510 laptop, using MATLAB as a framework for all camera functions. MATLAB's Image Acquisition Toolbox was employed to create real-time streakline images and movies, for characterisation of the flow type and structure, as well as acquiring individual frame-by-frame images. The Firewire camera was set to use a frame-rate of 3.75 frames/second for flow visualisation and it was found that the optimal streakline images were made via 11 captures with a 3 frame interval between each, giving effective streaks of roughly 8.8 seconds duration. The frame-by-frame images were then analysed using Correlation Image Velocimetry (CIV), as described by Fincham and Spedding (1997), for example. From this information, vector velocity field measurements of the flow were obtained by CIV and then further analysed, giving a detailed 
quantitative examination of the flow structure, including an azimuthal Fourier decomposition.

\subsubsection{Topographic Design}

The acrylic base topography was made up of interchangeable, radially-uniform flat segments combined with wavenumber-3 sinusoid components, with a minimum thickness of $10 \mathrm{~mm}$ and a maximum thickness of $60 \mathrm{~mm}$, an outer radius of $14.3 \mathrm{~cm}$ and an inner radius of $4.675 \mathrm{~cm}$ to allow a $2.5 \mathrm{~mm}$ gap from the sides of the annulus (to allow for expansion). In this way, the topography permits comparison, for example between flows over radially-varying topography, such as in Jin and Ghil's (1990) channel model, and those over radially-uniform topography. Since the topography in question was comprised of azimuthal segments, it allows for adaption to create an isolated ridge partial barrier. By combining flat base segments with a single peak from the wavenumber-3 base, a radially uniform barrier of sinusoidal zonal cross-section could be implemented, as illustrated in Figure 2, giving a blockage ratio of about $19 \%$ at the outer wall and $22 \%$ at the inner wall (due to the slope of the lid). This method allows for comparison of the effects of a curved wall with a sheer one (as utilised in previous works: e.g. Rayer et al. 1998), as well as between a partial barrier and a full-depth one (e.g. Wordsworth 2009), and finally between isolated and periodic topography (especially that of Marshall and Read 2015). The motivation for having a single ridge is that it serves to make obvious where (in terms of upstream or downstream of the peak) and how the flow interacts with the topography, both at the bottom level where the circulation is blocked, and at higher levels where it is unobstructed. Furthermore, Harlander et al. (2012) noted that a similar arrangement of topography would be wellsuited to the study of the transient evolution of downstream features of the flow structure in particular. As well as permitting the investigation of ocean-like topographic blocking, the curved ridge is arguably a more realistic simulation of general blocking structures in the atmosphere, and has rough parallels e.g. to the topographic impact of the Andes in the Southern Hemisphere. 
In addition, a transparent acrylic sloped lid was placed in contact with the fluid, as also used by Wordsworth et al. (2008) and Marshall and Read (2015), with a $22^{\circ}$ slope on the bottom of the lid and $3.5^{\circ}$ slope on the top, to correct for optical distortions at the set camera height. Under a sloped lid it was found to be easier to find resonant wave patterns, as the topographic beta-effect that is added by the sloping boundary to the fluid acts to slow down the azimuthal drift rate of the waves relative to the walls of the apparatus to near-zero. As such, stationary structures, and in turn topographic resonance phenomena, were found to occur more readily in the examined parameter space and at lower Taylor numbers. This was the same arrangement as utilised by Marshall and Read (2015) with a periodic wavenumber-3 topographic base.

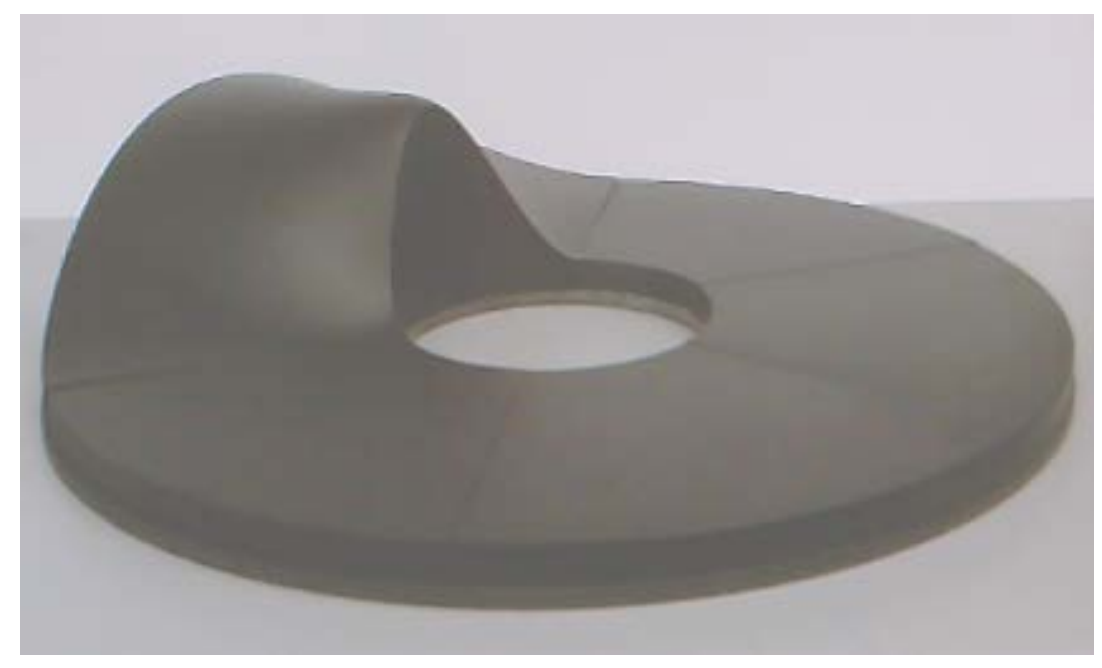

Figure 2: Bases arranged to produce a partial barrier

\subsection{Experimental Arrangement}

The results gathered were taken over a substantial range of parameter space, covering rotation rates from $0.4 \mathrm{rad} \mathrm{s}^{-1}$ to $2 \mathrm{rad} \mathrm{s}^{-1}$, at intervals of $0.2 \mathrm{rads}^{-1}$, and temperature differences from $2 \mathrm{~K}$ to $7 \mathrm{~K}$, at intervals of $1 \mathrm{~K}$. Each individual realisation was observed and recorded for 1100s, starting at 3600s after spin up to allow the flow to equilibrate.

The experiments were run with a lid in contact with the fluid that sloped downwards towards the centre, and with a heated inner cylinder and cooled outer cylinder (i.e. the reverse of more typical 
rotating annulus experiments, e.g. see Hide and Mason 1975), as used in a related study in the same apparatus (Marshall and Read 2015). This configuration was used so that the lid sloped in the same direction as the isotherms, thereby both increasing the instability of the freely evolving baroclinic waves and enabling their azimuthal phase propagation to become roughly stationary relative to the walls of the annulus, as discussed by Mason (1975). The latter was necessary in order to both promote a resonant response to the stationary quasi-wavenumber- 1 bottom topography as well as replicate the Coriolis effect from the Earth's curvature, which has a similar impact on atmospheric Rossby waves.

\section{Flow Regimes and Phenomena}

This section will focus on flow structures in the presence of a single isolated ridge, as a way of studying the effect of topographic partial barriers on the structure and behaviour of a baroclinic flow.

\subsection{Results and Analysis}

The locations of various types of flow regime encountered in the experiments are shown in the following section in the form of a two-parameter regime diagram in Figure 3. This regime diagram was compiled by identifying the dominant flow structure at each investigated point in parameter space. Both real-time streakline images and sequences of horizontal vector velocity field maps created by CIV were used to identify the principal flow regimes. 


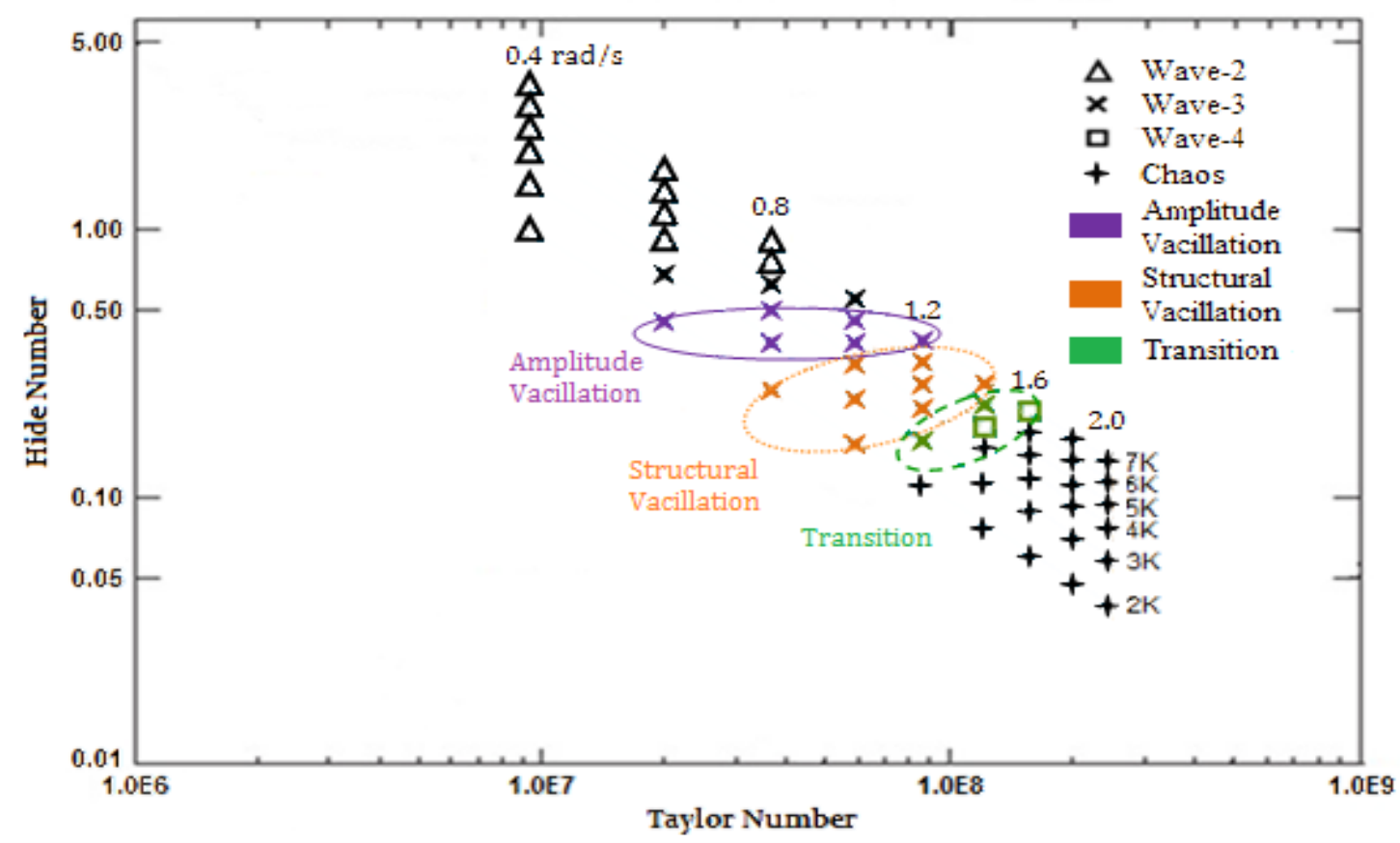

Figure 3: Regime diagram showing the locations and dominant flow characteristics encountered in the conducted Partial Barrier experiments. Moving diagonally from upper left to lower right permits lines of constant temperature difference, as indicated.

The results shown in the regime diagram in Figure 3 tend to fall into either of two major categories: an 'interference' region, occurring at larger Hide numbers, where the impact of the stationary wave disturbance forced by the topography is strongly observable throughout the flow, superposed upon and 'interfering' with any drifting components; and an 'irregular' region, at lower Hide numbers, where the flow is erratic in time and space. Within the 'interference' regime baroclinic waves can be observed, and the circulation never becomes a purely axisymmetric flow (almost certainly due to the asymmetrical topography). Instead the flow always exhibits a drifting wave structure, transitioning from a dominant wavenumber-2 to wavenumber-3, and with occasional transitions to wavenumber-4, as the rotation rate increases. Compared to experiments with pure wavenumber-3 bottom topography, such as investigated by Read and Risch (2011), Marshall and Read (2015) and Risch and Read (2015), amplitude and/or structural vacillations were encountered in similar areas of parameter space, but some points, denoted in Figure 3 as the dashed (green online) Transition region, exhibit a greatly increased number of apparent transitions between wavenumbers over time. In these 
flows, which lie near the 'irregular' region, a given dominant wavenumber is only foremost for part of the time, with other wavenumbers irregularly dominating the structure. This is especially common in exchanges between wavenumber- 3 and wavenumber- 4 , as those are the wavenumbers most often encountered before fully erratic behaviour takes over. Typically, the transitions are between adjacent wavenumbers, so that wavenumber-2 may transition to wavenumber- 1 or wavenumber-3, and wavenumber-3 may transition to wavenumber-2 or wavenumber-4. This could suggest a lowdimensional wavenumber competition via wave-zonal flow nonlinear interactions (see e.g. Read et al. 1992). Harmonics and sidebands with wavenumbers higher than 4 were observed, but found to never dominate the flow structure, which is consistent with previous studies in the same annulus with periodic topography (see Marshall and Read 2015).

\subsubsection{Time-Dependent 'Interference' Flow Structure}

In addition to the above, it can be noted that the drifting waves are modified as they encounter and cross the topography, creating temporary flow structures at all levels of the experiment in the vertical direction. To illustrate this effect, an example of the sequence of time variations of the flow structure within this region during the Partial Barriers experiments is presented in Figure 4. 
a.)

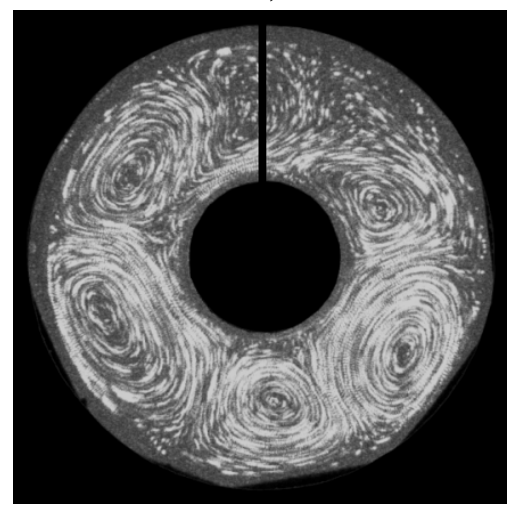

c.)

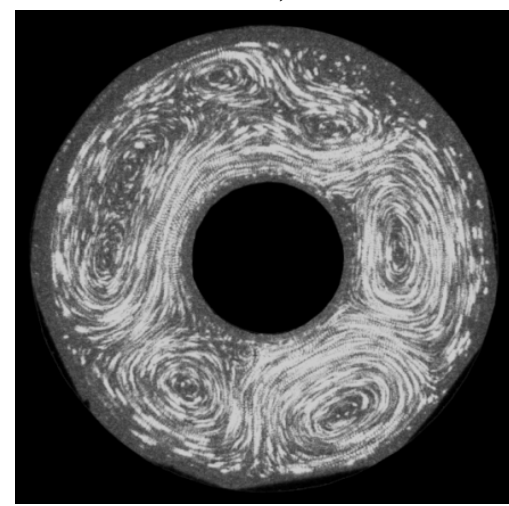

e.)

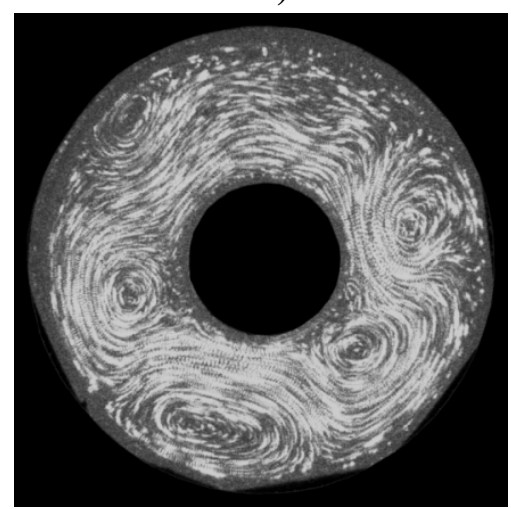

g.)

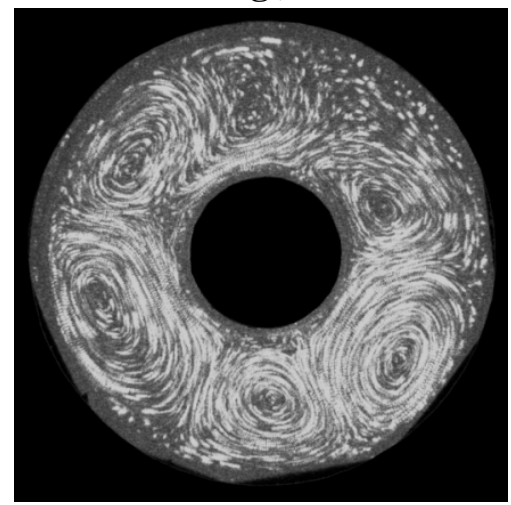

b.)

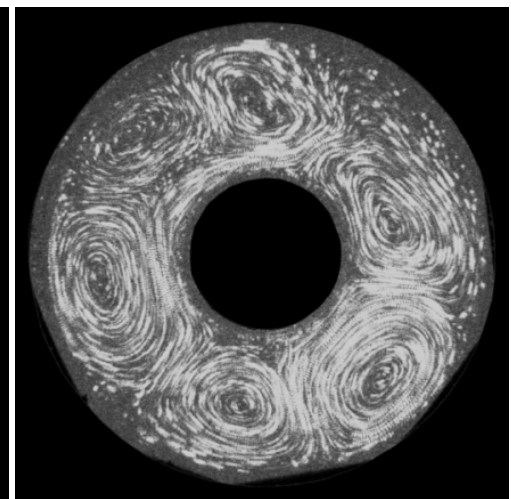

d.)

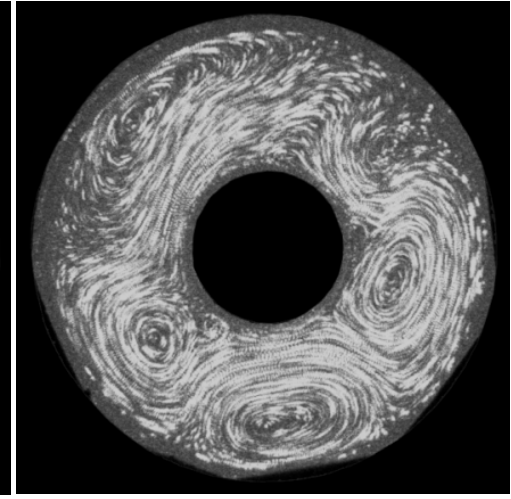

f.)

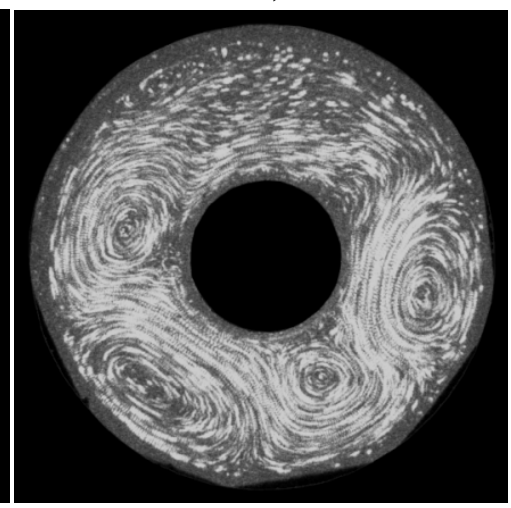

h.)

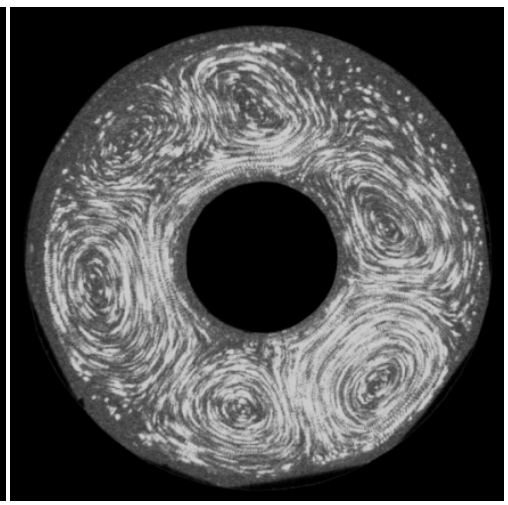

Figure 4: Streakline series for Partial Barriers Experiments at Level 3 (mid-depth), $\Omega=0.8$ rads $^{-1}, \Delta T=4 K$, $\mathcal{T}=3.635 \times 10^{7}, \Theta_{\mathrm{T}}=0.494$, a.) $t=3650 \mathrm{~s}$, b.) $t=3680 \mathrm{~s}$, c.) $t=3710 \mathrm{~s}$, d.) $t=3740 \mathrm{~s}$, e.) $t=3770 \mathrm{~s}$, f.) $t=3800 \mathrm{~s}, \mathrm{~g}$.) $t=3830 \mathrm{~s}, \mathrm{~h}$.) $t=3860 \mathrm{~s}$. A black line denotes the topographic peak in (a). 
For clarity, an indication of where the peak of the topography lies has been added to Figure 4a, so that the main aspects of the 'interference' regime can be observed. The flow in (a) starts off as a clockwise-drifting wavenumber-3, but during (b) one of the cyclonic eddies (at the upper left of the image) encounters the topography and weakens until it disappears. At the same time, the weak anticyclonic eddy directly above the topography strengthens. In (c) this anticyclonic eddy joins up with a second further upstream (anticlockwise). The flow then appears to be a skewed wavenumber- 2 which steadily continues drifting throughout (d) and (e). This continues until (f), where the previously disappeared cyclonic eddy seems to begin to reappear on the other (downstream) side of the topography - the flow now resembling a wavenumber-3, but still remaining skewed. By (g), the flow has drifted far enough that the original wavenumber-3 structure has returned, and this then continues until the next eddy encounters the topography, as in (h), whereby the cycle repeats itself. The example given in Figure 4 shows the flow at mid-depth, but similar effects can be found at all levels except near the bottom, which is physically blocked by the base topography and will be discussed later. At lower rotation rates, the dominant component is a wavenumber-2, and so when the waves disappear when encountering the topography the flow resembles a skewed wavenumber-1. This would appear to be an example of the topographic effect of jet shifting, also known as jet deflection, as discussed by Tian et al. (2001). This jet-shifting phenomenon was also noted in the experiments with both partial vertical and radial barriers of Harlander et al. (2012), who found similar transitions from wavenumber-3 to a skewed wavenumber2 as the waves crossed the topographic peak.

To illustrate the 'interference' regime flow structures over a longer timescale than the image sequence above, Figure 5 shows an azimuth-time Hovmöller diagram of the radial velocity for every point on a complete azimuthal circle at mid-radius, for the same parameters as in Figure 4 over the entire 1100s run. Azimuthal position is given in degrees (where positive is anticlockwise), and wherein the centre of the topographic peak occurs at about $100^{\circ}$. 

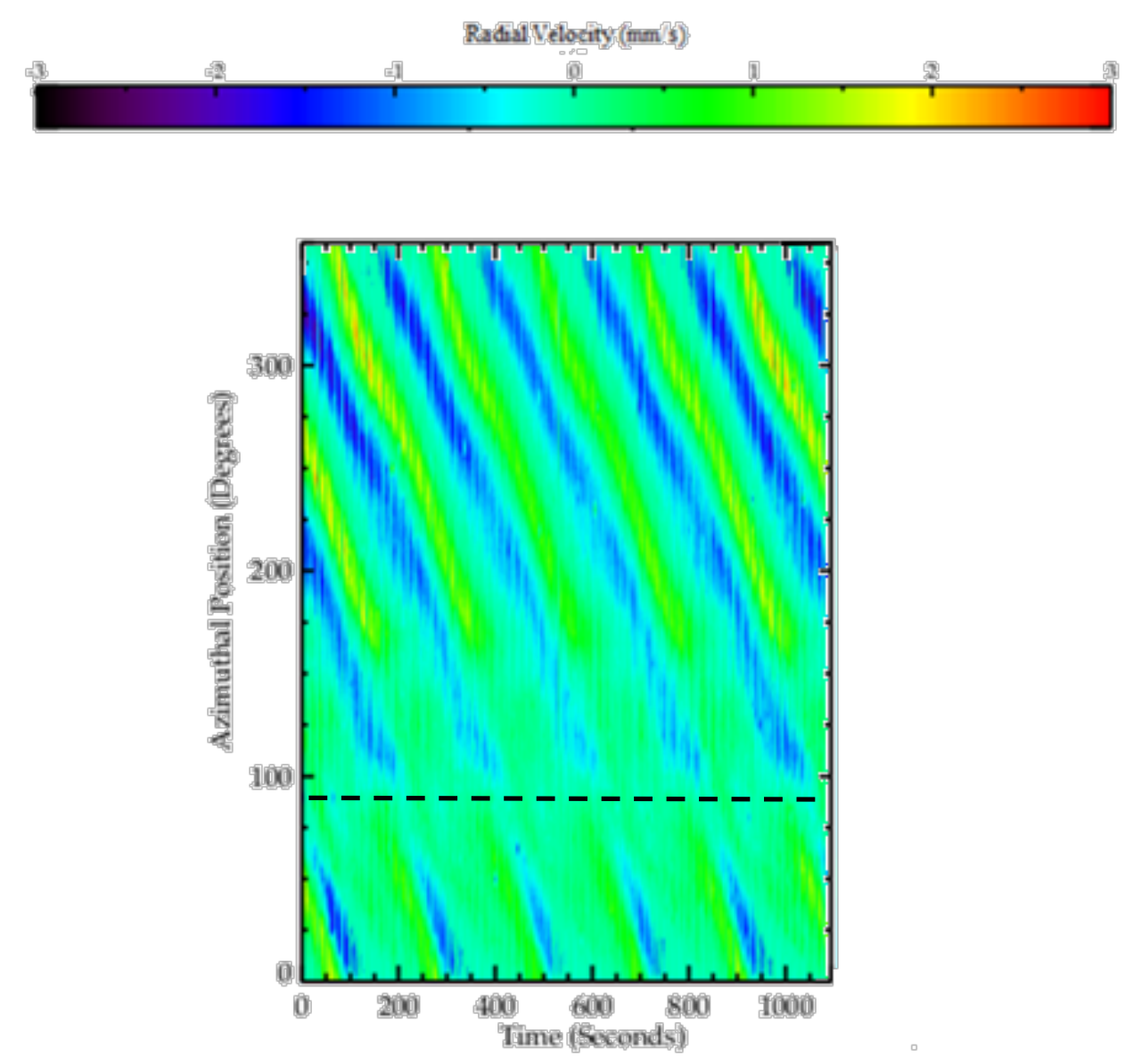

Figure 5: Radial velocity as a function of time and azimuthal position at mid-radius for Level 3 (mid-depth), $\Omega=0.8 \mathrm{rads}^{-1}, \Delta T=4 K, \mathcal{T}=3.635 \times 10^{7}, \Theta_{\mathrm{T}}=0.494$. The black dashed line denotes the topographic peak.

Figure 5 shows the continuously clockwise drifting wavenumber-3 structure of the flow throughout this example of the 'Interference' region of parameter space, becoming more negative in azimuthal position over time. The impact upon an eddy as it encounters the topography is also highlighted, which begins at around an azimuthal position of $150^{\circ}$ and ends at around $50^{\circ}$. At the onset point, the drifting eddy can be observed to reduce in strength greatly, becoming difficult to discern from the background flow. This is equivalent to the apparent visual disappearance of part of the wavenumber3 structure as it encounters the topographic peak as shown in Figure 4. The phase of the drifting eddy is also notably modified as it crosses the topographic peak. In addition, Figure 5 suggests that the eddy that disappears when crossing the topography is indeed the same eddy that reappears once the flow has moved on, due to the unbroken peak or trough connecting the drifting flow on either side of the 
topography. Finally, it appears that the eddy is strongest when on the opposite side of the annulus to the topography, at about $300^{\circ}$ in Figure 5.

\subsubsection{Time-Dependent 'Irregular' Flow Structure}

An 'irregular' region appears abruptly in the mapped parameter space, and at much lower rotation rates and temperature differences than would be expected for such an erratic flow without the presence of asymmetrical bottom topography. To illustrate further some of the unusual properties of this regime, a brief example of the flow structure is given in Figure 6, which highlights some of the notable features of this 'irregular' regime. Firstly, (a) shows what is either a skewed wavenumber-3, or a wavenumber-4 in which the eddy above the topography is missing. This lack of activity above the topography continues in (b), where what appears to be a skewed wavenumber-4 can be seen, but this quickly dissipates. Until this point, the flow structure has exhibited noticeable fine-scale features, such as small, irregularly-spaced eddies. But in (c) these features are dominant in the circulation. Starting at (d), it can be noted that the eddies downstream of the topography (clockwise) are generally larger in linear size than those upstream of it. In addition, by (e) and (f) a large persistent stationary eddy has appeared directly above the topography. Throughout all these images, however, a large eddy or a jet can be seen just upstream of the topographic peak - the occurrence of this structure at this location seems to be a constant feature of the flow, unlike the smaller eddies that appear and disappear irregularly over time. 
a.)

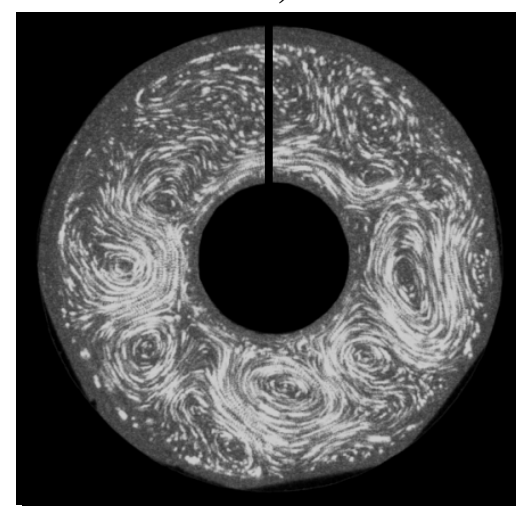

c.)

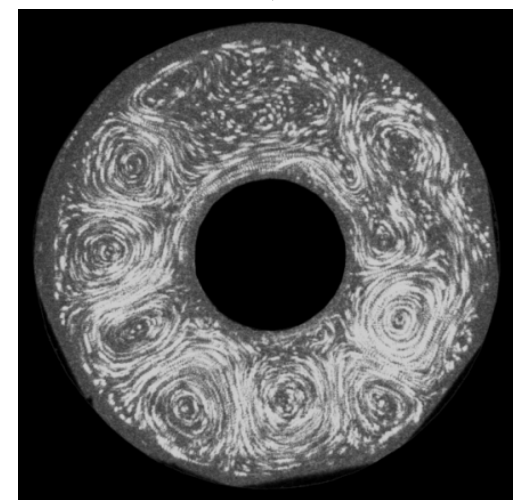

e.)

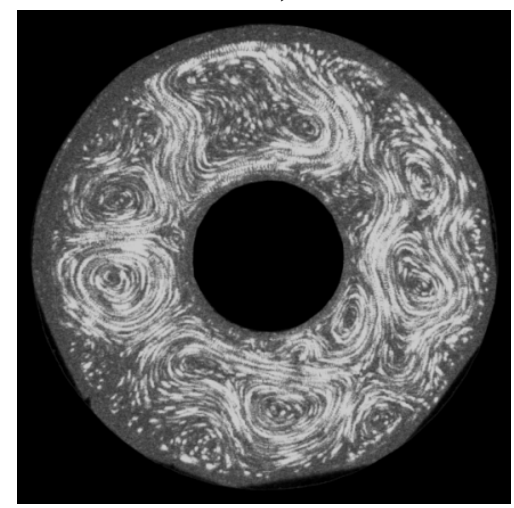

b.)

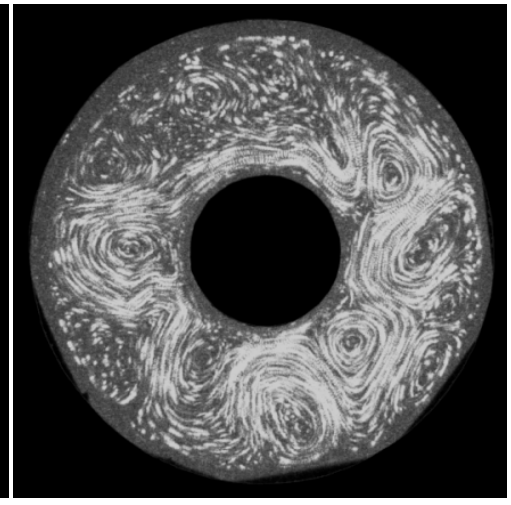

d.)

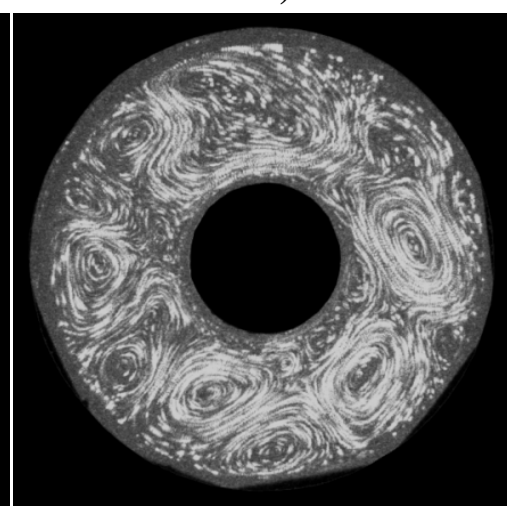

f.)

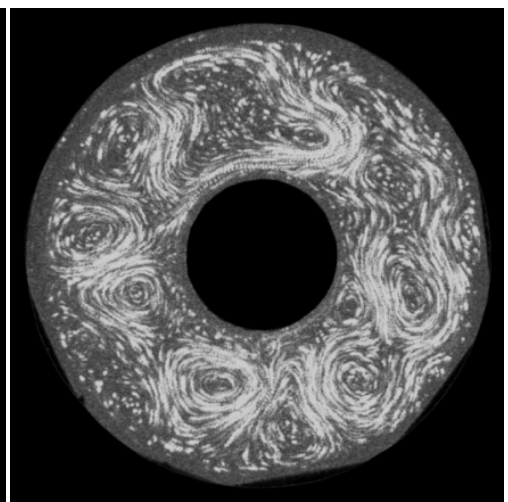

Figure 6: Streakline series for Level 3 (mid-depth), $\Omega=1.6 \mathrm{rads}^{-1}, \Delta T=4 K, \mathcal{T}=1.454 \times 10^{8}, \Theta_{\mathrm{T}}=0.124$, a.) $t=3600 \mathrm{~s}$, b.) $t=3680 \mathrm{~s}$, c.) $t=3760 \mathrm{~s}$, d.) $t=3840 \mathrm{~s}$, e.) $t=3920 \mathrm{~s}$, f.) $t=4000 \mathrm{~s}$. A black line denotes the topographic peak in (a).

The most notable aspect of this 'irregular' regime, however, is that it appears over a very small range in rotation rate, since readings were as close as $0.2 \mathrm{rads}^{-1}$ apart and neither flow shows any evidence of the structure of the other, apart from limited transitions between wavenumbers. Furthermore, each flow regime was found to develop directly from the other, forming simply by incrementing the rotation rate (either increasing or decreasing) and without requiring a stop of the 
apparatus. This would appear to imply a discontinuous transition or bifurcation between the 'interference' and 'irregular' regions. In order to explore this in more detail, further runs were carried out at smaller intervals of $0.05 \mathrm{rads}^{-1}$, and yet chaotic irregular behaviour was still found to appear suddenly from a previously ordered and symmetrical flow, occurring, for example, between 1.2 rads $^{-1}$ and 1.25 rads $^{-1}$ when at a temperature difference of $4 \mathrm{~K}$ (a change equal to increment values of $\Delta \mathcal{T}=$ $6.958 \times 10^{6}$ and $\Delta \Theta_{\mathrm{T}}=1.722 \times 10^{-2}$, and percentage variations of $\Delta \mathcal{T}=8.5 \%$ and $\Delta \Theta_{\mathrm{T}}=4 \%$ ). This is surprising for such a dramatic change since, for example, the transition towards irregular flow without asymmetrical bottom topography is typically preceded by steadily increasing levels of vacillation and small-scale dynamical noise, such as was noted by Marshall and Read (2015). In addition, hysteresis, found to be negligible in Marshall and Read's experiments with a wavenumber-3 base, was observed throughout this small area of parameter space, in relation to the beginning of the 'irregular' regime. For example, the result at a rotation rate of 1.25 rads $^{-1}$ and with a temperature difference of $4 \mathrm{~K}$ was found to have an 'irregular' flow structure (appearing as in Figure 6) when the rotation rate was increased, as discussed above, but an 'interference’ structure (appearing as in Figure 4) when the annulus was spun up from rest. As such, it appears that the initial conditions of the fluid have an effect on where the transition between these regimes occurs, with the 'irregular' regime occurring at lower Taylor numbers during a scan with increasing rotation rate.

To illustrate the 'irregular' regime flow structure over a longer timescale than the image sequence in Figure 6, Figure 7 shows a Hovmöller plot of the radial velocity for every point on a complete azimuthal circle at mid-radius, for the same parameters over the entire 1100s run. Once again, azimuthal position is given in degrees, where positive is anticlockwise, and wherein the centre of the topographic peak occurs at an azimuth of about $100^{\circ}$. 

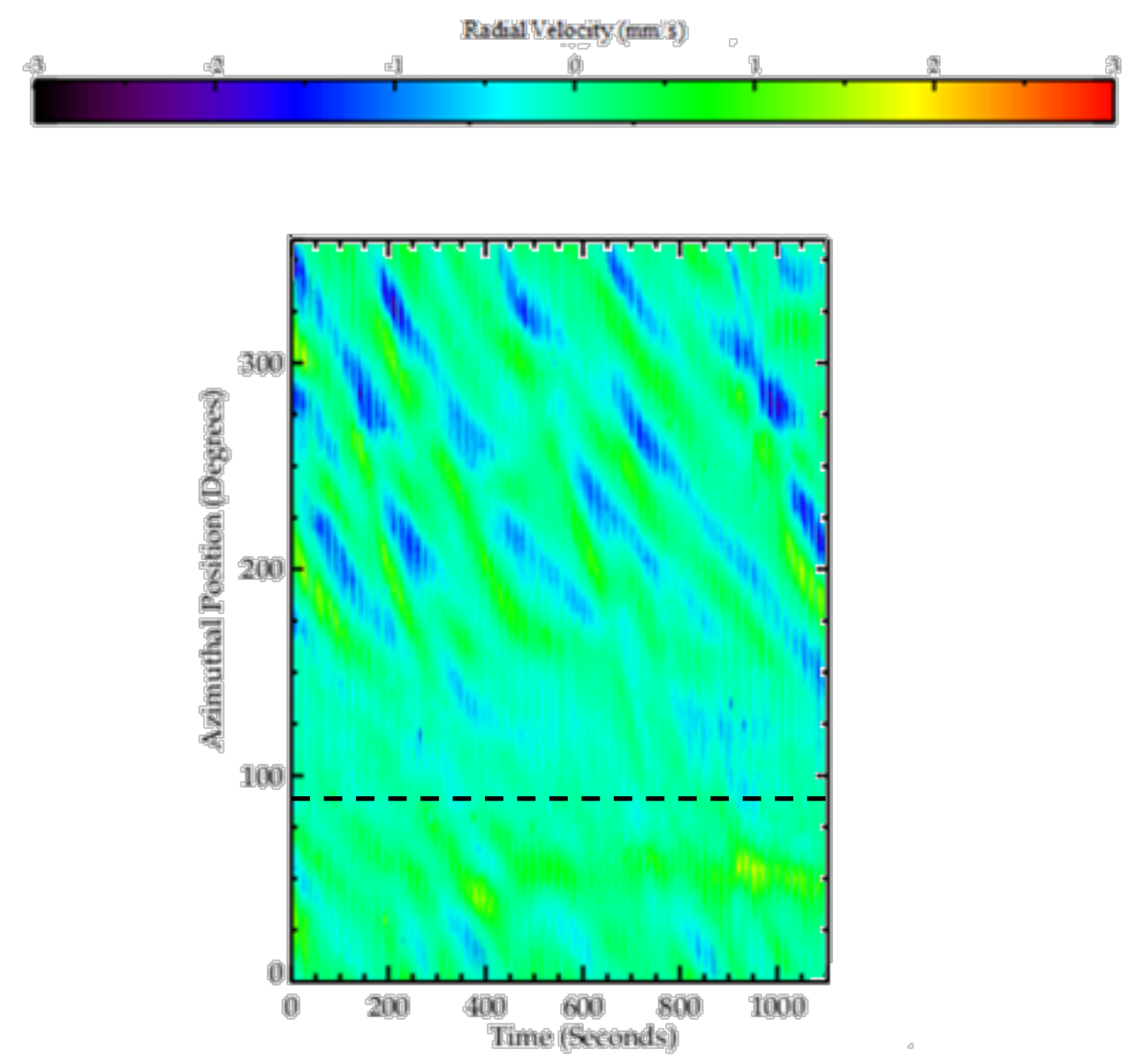

Figure 7: Radial velocity over time against azimuthal position at mid-radius for Level 3 (mid-depth) at $\Omega=1.6$ rads $^{-1}, \Delta T=4 K, \mathcal{T}=1.454 \times 10^{8}, \Theta_{\mathrm{T}}=0.124$. The black dashed line denotes the topographic peak.

Figure 7 shows a far more erratic flow variation throughout the entire run than in the 'interference' regime case in Figure 5, but also shows that the flow is not purely random in nature. Some clockwise drifting wave structures representing several different wavenumber components can be observed, especially far from the mechanical topography, at around an azimuthal position of $300^{\circ}$ in (a). These different wavenumber components have varying drifting rates and often overlap each other, contributing to the erratic appearance of the flow. Nearer the topography, however, the flow structure becomes too erratic to be able to observe any drifting flow, with only the impact of the topographic peak (as a weakening and then reappearance of the waves) being visible. 


\subsection{Time-Averaged Flow Structure}

Taking a time-average of the flow structure over the period of the drifting waves (taken from Figure 4 to be about 200s) has the effect of removing the drifting wave elements and leaving only the stationary components. To illustrate this, Figure 8 gives an example of the time-averaged flow for the 'interference' regime using a kinetic energy map for the parameters given in Figure 5. This map was found to be reasonably equivalent regardless of the specific flow data that was time-averaged.

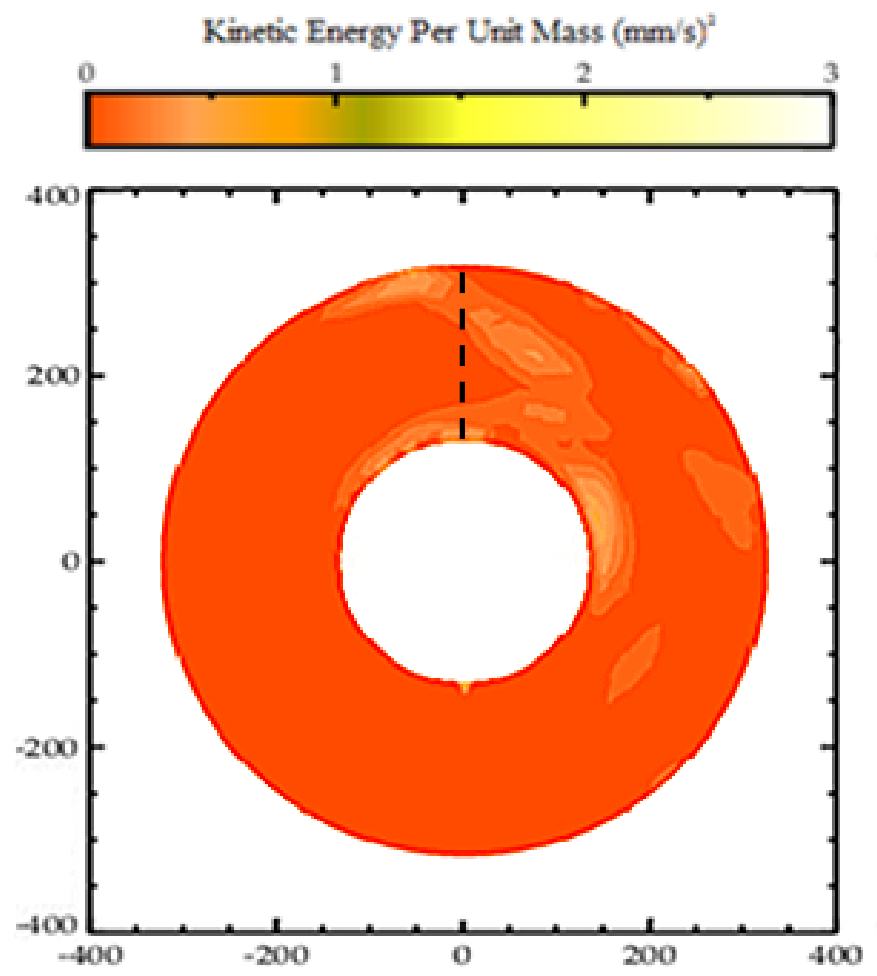

Figure 8: Kinetic energy averaged over 200s for Level 3 (mid-depth) at $\Omega=0.8 \mathrm{rads}^{-1}, \Delta T=4 K, \mathcal{T}=$ $3.635 \times 10^{7}, \Theta_{\mathrm{T}}=0.494$. The black dashed line denotes the topographic peak.

As Figure 8 illustrates, with the drifting wave components removed by the time-averaging, a great deal of the flow structure is removed. By combining the averaged velocity components into a kinetic energy map to illustrate the total flow structure, only a single notable persistent jet remains, forming a weak stationary vortex directly above the bottom topography. This is evidence for a pressure drop as the flow crosses the topographic peak (since the radial and azimuthal velocities show that the 
relative vorticity is cyclonic), and also highlights the relative lack of stationary wave elements except in the vicinity of the topography. In addition, the stationary "imaginary cylinder" occurring in the flow directly above the actual topography suggests an analogy with a Taylor column (see Taylor 1923).

Similarly, Figure 9 shows a time-average of the flow structure over the period of the drifting waves for the 'irregular' regime, using a kinetic energy map computed at the parameters given in Figure 7.

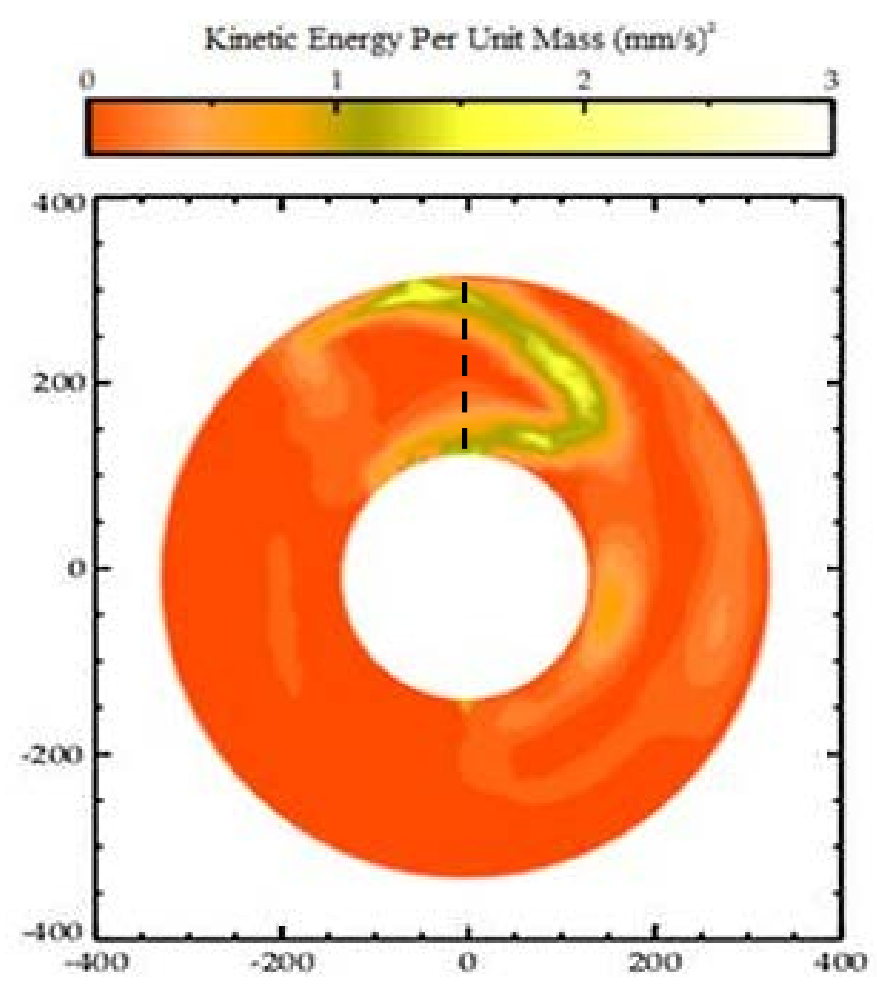

Figure 9: Kinetic energy averaged over 200s for Level 3 (mid-depth) at $\Omega=1.6 \mathrm{rads}^{-1}, \Delta T=4 K, \mathcal{T}=$ $1.454 \times 10^{8}, \Theta_{\mathrm{T}}=0.124$. The black dashed line denotes the topographic peak.

Figure 9 demonstrates that the 'irregular' region has a very similar time-averaged structure to the 'interference' region, with weak stationary elements except for a single notable persistent jet occurring directly above the bottom topography. The jet and corresponding pressure drop as the flow crosses the topographic peak are significantly larger in amplitude for the 'irregular' regime, however, and a downstream (clockwise) wake can also be noted. This suggests that the impact of the topography 
upon the flow becomes greater as Taylor number is increased - this is likely associated with the Charney-Drazin theorem (described in detail in Charney and Drazin 1961), which notes that vertical propagation of stationary wave features forced by the bottom boundary will occur more readily under a higher rotational forcing (and hence larger beta effects).

\subsection{Bottom Flow Structure}

At the lowest vertical level observed, the horizontal flow is physically blocked by the topographic peak of the base. The flow is therefore forced to create an azimuthally-trapped structure (though fluid may be driven upwards over the ridge), different to that found at the higher, unobstructed, levels. Figure 10 gives an example of this flow, using a light level $8 \mathrm{~mm}$ above the peak of the topography, at a point in parameter space close to the boundary between the 'interference' and ‘irregular’ regimes. 
a.)

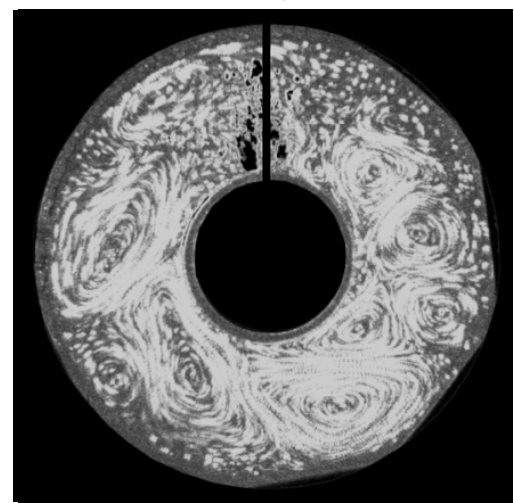

c.)

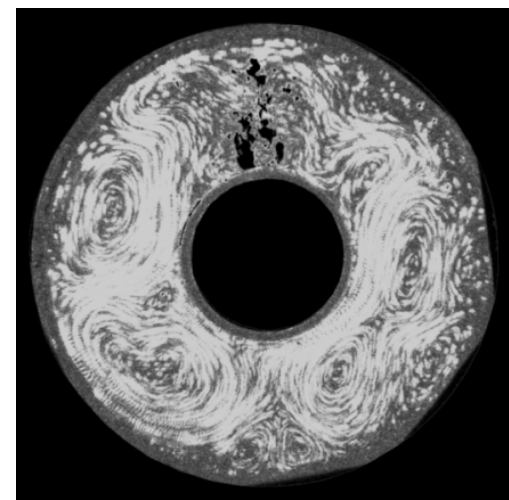

b.)

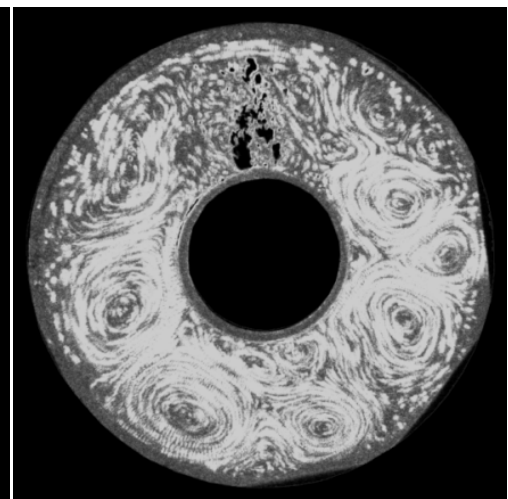

d.)

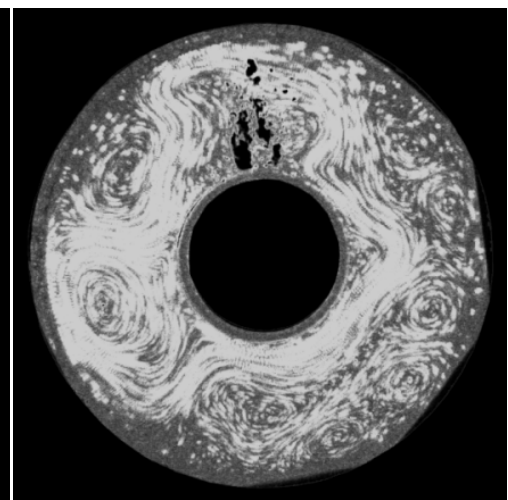

e.)

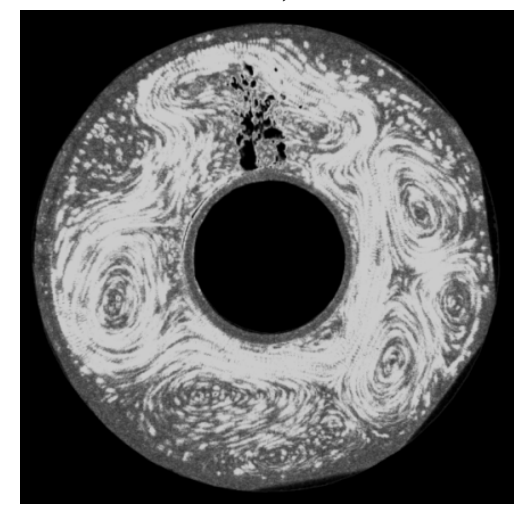

Figure 10: Streakline series of images at Level 5 (bottom), $\Omega=1.1 \mathrm{rads}^{-1}, \Delta T=4 K, \mathcal{T}=6.873 \times 10^{7}, \Theta_{\mathrm{T}}=$ 0.261, a.) $t=3600 \mathrm{~s}$, b.) $t=3680 \mathrm{~s}$, c.) $t=3760 \mathrm{~s}$, d.) $t=3840 \mathrm{~s}$, e.) $t=3920 \mathrm{~s}$. A black line denotes the topographic peak in (a).

Figure 10 shows the main aspects of the trapped flow, and highlights the location of the topographic peak via a black line. At this peak, particles have settled on the base, creating a black shadow close to the 12 o'clock position in each frame. Whilst the flow structure in (a) appears to be irregular at first, it can be noted that the eddies in the 'blocked' region downstream (clockwise) of the topography are smaller than those in the 'unblocked' region upstream of the topography. This continues 
through (b) until (c), when the upstream cyclonic eddies grow in size to form a skewed wavenumber-2 structure, with a jet that wraps around the topographic peak. In (d) and (e) this wavenumber-2 pattern becomes more skewed as the upstream eddy slowly drifts, but the jet above the peak does not. Due to this persistent azimuthally-trapped wave, as well as the clear asymmetry between the smaller and larger eddies seen throughout, the flow has many of the features of the 'irregular' regime, as shown in Figure 6. If the wavenumber-2 collapses, which was occasionally found to occur irregularly, the flow returned to the arrangement of (a). This occurrence was universal for all parameters, though the flow in the 'interference' regime favours the structure found in (c), (d) and (e), and the flow in the 'irregular' regime shows more of the smaller eddies of (a) and (b). Once again, this is similar to the results found by Harlander et al. (2012), who observed a trapped wave and smaller downstream eddies. However, in contrast to their results, the trapped wave in our experiments was found at mid-depth and was surrounded by a large jet similar to that of Wordsworth's (2009) ocean basin experiments. These differences are likely due to Harlander et al.’s addition of the partial radial barrier, which did not span the gap in the channel in either the radial or vertical directions.

\section{Time Variations in Amplitude and Phase}

To allow further understanding of the observed oscillations, an azimuthal Fourier analysis of the wavenumber structure in terms of their amplitudes and phases was carried out for every time step. Combining these analyses allows the evolution of the flow over time to be observed. After the zonal background mean flow was removed, azimuthal and radial velocity components were calculated at a quarter of the radial distance between the inner wall and the outer wall (closer to the inner wall) and at mid-radius respectively, chosen to avoid nodes in the radial direction. 


\section{1. 'Interference' Regime Fourier Analysis}

Figure 11 shows an example of the time variations of the amplitude of the first four wavenumbers, for typical flows in the 'interference' regime, far away from the boundary with the ‘irregular' regime, for the same parameters as used in Figure 5.
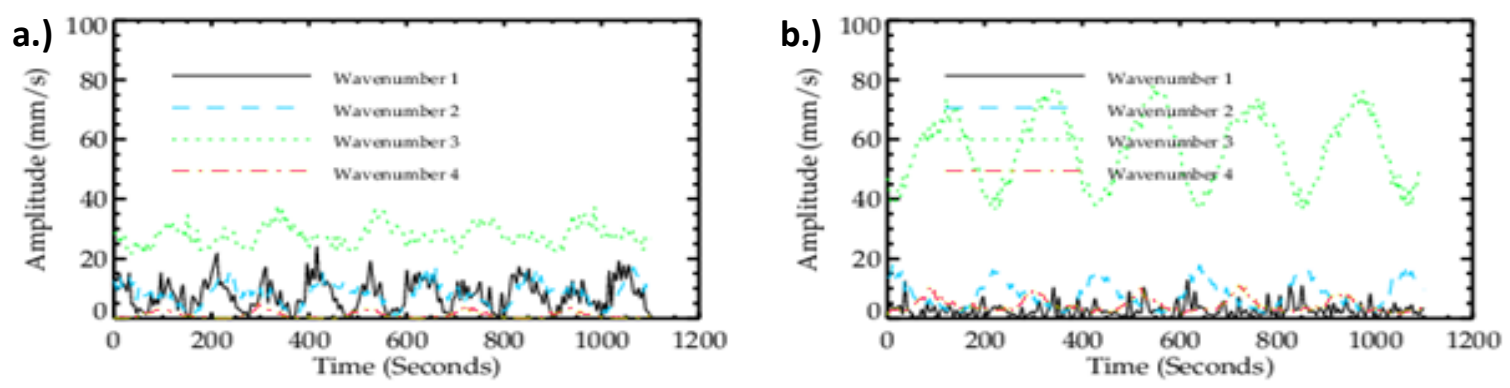

Figure 11: Velocity amplitude for wavenumbers 1-4 at Level 3 (mid-depth) at $\Omega=0.8 \mathrm{rads}^{-1}, \Delta T=4 K, \mathcal{T}=$ $3.635 \times 10^{7}, \Theta_{\mathrm{T}}=0.494 ;$ a.) azimuthal velocity, b.) radial velocity.

As can be seen in Figure 11, the flow in the 'interference' region is primarily comprised of wavenumber-1, wavenumber-2 and wavenumber-3 components, with the latter strongly dominant throughout. The amplitudes can be seen to have a regular oscillatory nature, with the peaks of the wavenumber-2 component (and the wavenumber-1 component, in the azimuthal velocity case) matching the troughs of the wavenumber-3 component, on a timescale of roughly 180s. As such, the transitions between the dominant wavenumber- 2 and wavenumber-3 components would seem to correspond to the interactions with the topography shown in Figure 4. These have a period of about 180s, visually appearing as transitions between a temporary skewed wavenumber-2 structure and a more regular wavenumber-3 structure as the waves cross the topographic peak.

By examining the phases over time of the first ten wavenumbers of the flow at the same parameters as in Figure 11, most of the wave components were found to be steadily drifting. As the flow with mechanical topography at this location in parameter space is dominated by wavenumber-1, wavenumber-2 and wavenumber-3 components, Figure 12 shows the phase of these notable wavenumbers. 

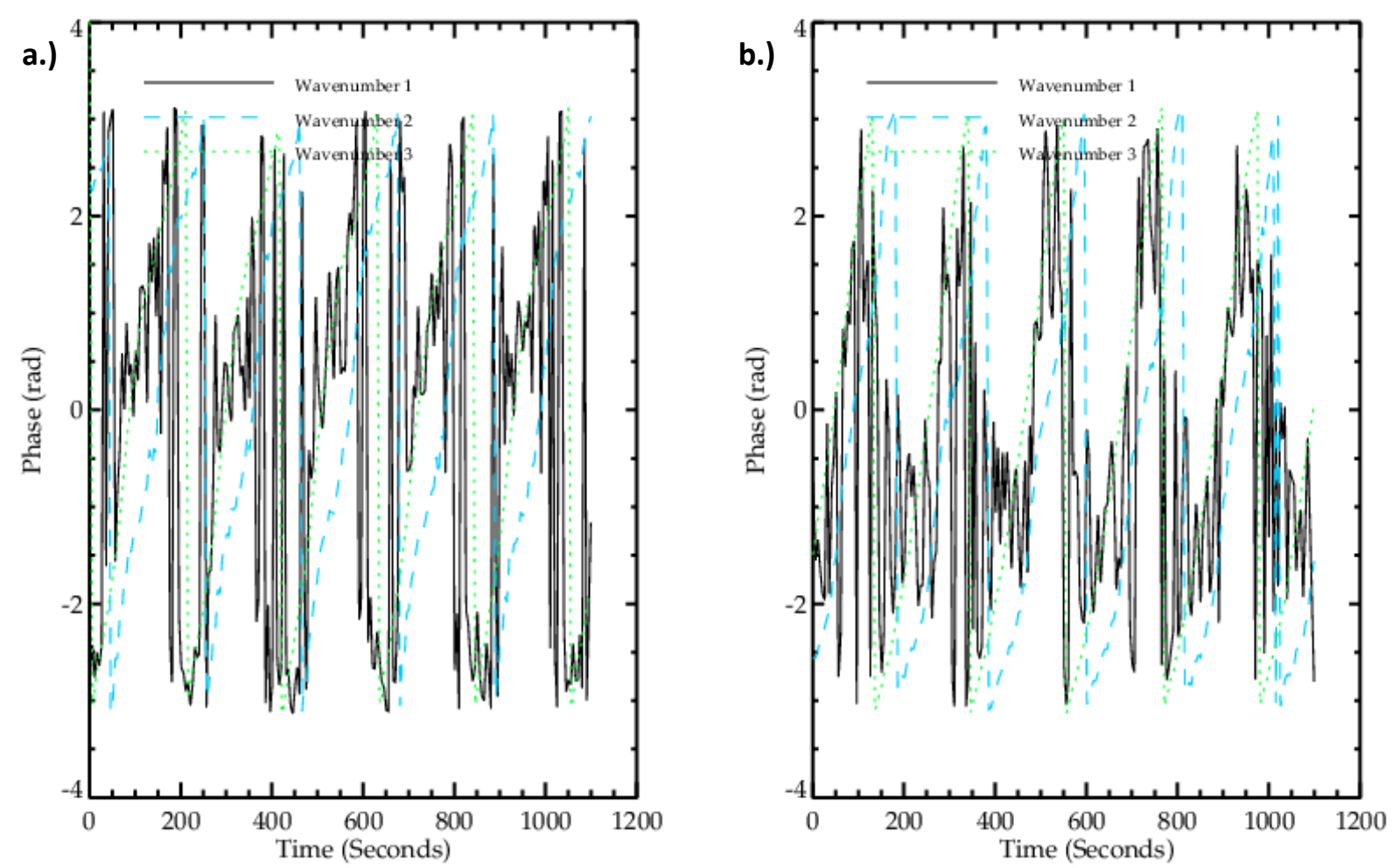

Figure 12: Velocity phase at Level 3 (mid-depth) at $\Omega=0.8 \mathrm{rads}^{-1}, \Delta T=4 K, \mathcal{T}=3.635 \times 10^{7}, \Theta_{\mathrm{T}}=0.494$; a.) azimuthal velocity for wavenumbers $1-3, b$.) radial velocity for wavenumbers 1-3.

Both (a) and (b) of Figure 12 illustrate that all of the wave components are constantly drifting with the same average frequency, despite the fact that the amplitude variations of the wavenumber-2 and wavenumber-3 components are in antiphase, and that the phase velocities would be expected, in the absence of nonlinear interactions, to follow a natural dispersion relation qualitatively consistent with the classical Rossby wave dispersion relation. It is also surprising that the wavenumber- 1 component at mid-height is not predominantly stationary, despite the strong wavenumber-1 element forced by the topographic base. Evidence of this forcing can still be observed, however, occurring as a regular 'flattening' of the wavenumber-1 component's slope roughly every 180s (i.e. the drift period) at about 0.5 rad for azimuthal velocity and about -1 rad for radial velocity (this feature is more apparent in the radial case). This 'flattening' is not seen for the wavenumber-2 or wavenumber-3 components, demonstrating that there must be both drifting and stationary elements to the wavenumber-1 component. The streakline images of the flow near the bottom of the annulus showed that there was an azimuthally- 
trapped stationary wave feature just upstream of the topography, so the 'interference' flow structure is therefore likely an interaction between the effects of the bottom-trapped stationary wave (i.e. the stationary wavenumber-1 element) and the drifting waves at mid-height (and above), thereby extending the reach of the topography far beyond its physical dimensions. During periods where the two are out of phase, they constructively interfere, creating a wavenumber-3 flow. In periods where they are in phase, they destructively interfere, creating the visually-skewed wavenumber- 2 flow. As the period of this interaction is again found to be roughly 180s, this suggests that the drifting and stationary waves become in phase when the one of the flow's drifting wave structures encounters the topography and interacts with it, causing the wave structure to temporarily disappear.

For further investigation, Figure 13 shows the variations of the radially-averaged kinetic energy of the first four wavenumbers over time, for the same flow case as in Figure 12.

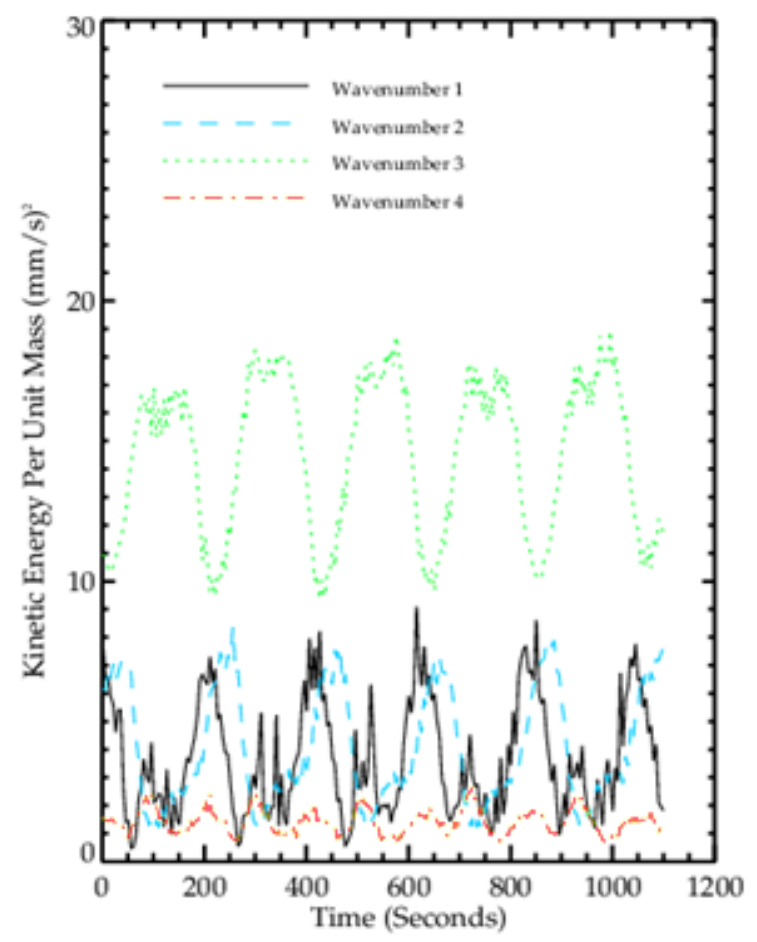

Figure 13: Radially-averaged kinetic energy for wavenumbers 1-4 at Level 3 (mid-depth) at $\Omega=0.8$ rads $^{-1}$, $\Delta T=4 K, \mathcal{T}=3.635 \times 10^{7}, \Theta_{\mathrm{T}}=0.494$. 
Figure 13 confirms what was just described, with the kinetic energy of the flow being transferred back and forth with a period of about 180s between the wavenumber-3 component and the wavenumber-1 and wavenumber-2 components, with the amplitude peaks of one wavenumber aligning with the troughs of the other. Indeed, the relationship between the two oscillations is even easier to discern in the graph of kinetic energy.

A contrast can be also made between the time variations of the kinetic energy of the dominant wavenumber-3 component and the kinetic energy of the zonal mean flow. At mid-depth, this zonal mean component is found to fluctuate periodically in direction from positive to negative and vice versa. This is presumably due to the zonal mean flow being acted upon by both the topographically-forced stationary waves from the bottom, appearing to dictate a 180s transitional oscillation associated with the drift period of the freely travelling baroclinic waves from the top and middle of the tank. Hence, Figure 14 shows the variation over time of the kinetic energy of the zonal mean from the same points in parameter space as in Figure 13, but at the higher Level 2. A superposition of the wavenumber-3 kinetic energy time-series is also provided for comparison.

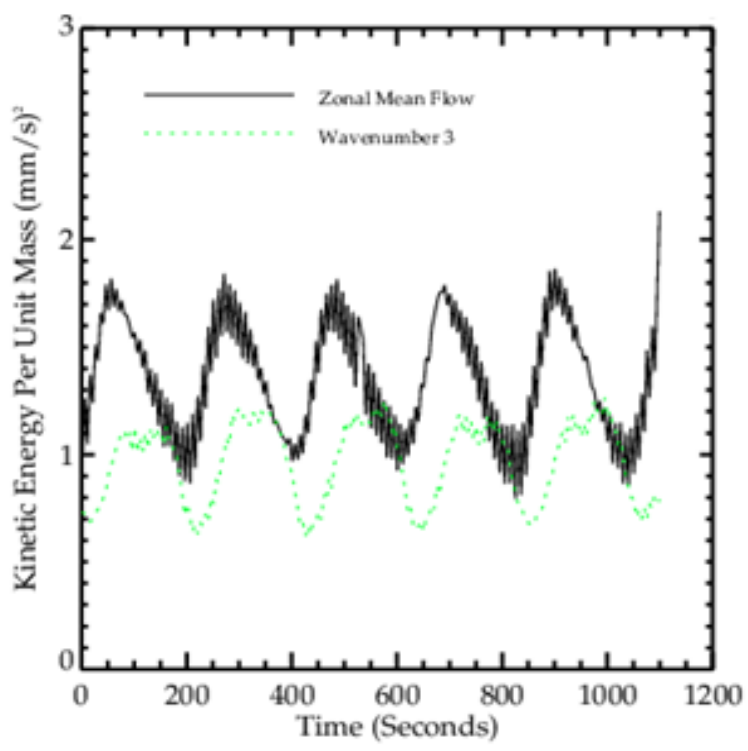

Figure 14: Radially-averaged kinetic energy of zonal mean flow (black) at Level 2 with wavenumber-3 total kinetic energy (green, not to scale) as comparison, at $\Omega=0.8 \mathrm{rads}^{-1}, \Delta T=4 K, \mathcal{T}=3.635 \times 10^{7}, \Theta_{\mathrm{T}}=0.494$. 
Figure 14 shows that the kinetic energy of the zonal mean flow for both cases oscillates effectively in quadrature with the kinetic energy of the dominant mode, with the zonal mean kinetic energy peaks occurring where the wavenumber-3 kinetic energy for mechanical or wavenumber-1 kinetic energy for thermal is changing most rapidly, and vice versa. This is consistent with strong nonlinear wave-zonal flow interactions and an exchange of energy between the waves and the mean zonal flow. These results are comparable with those found in the presence of pure wavenumber-3 topography, such as used in the experiments of Marshall and Read (2015). They also resemble the findings of Charney and Strauss (1980), who noted an oscillating transfer of potential energy between eddies and the zonal mean flow in their baroclinic model, leading to topographically-forced stationary waves and multiple equilibria. The large peak in zonal mean flow at 1100 s is an anomaly, caused by the experiment being stopped fractionally too early.

\section{2. 'Irregular' Regime Fourier Analysis}

For contrast with the 'interference' regime, Figure 15 gives the time variations of the amplitude of selected wavenumbers of typical flows in the 'irregular' regime, fairly distant from the region boundary in parameter space, for the parameters used in Figure 7.
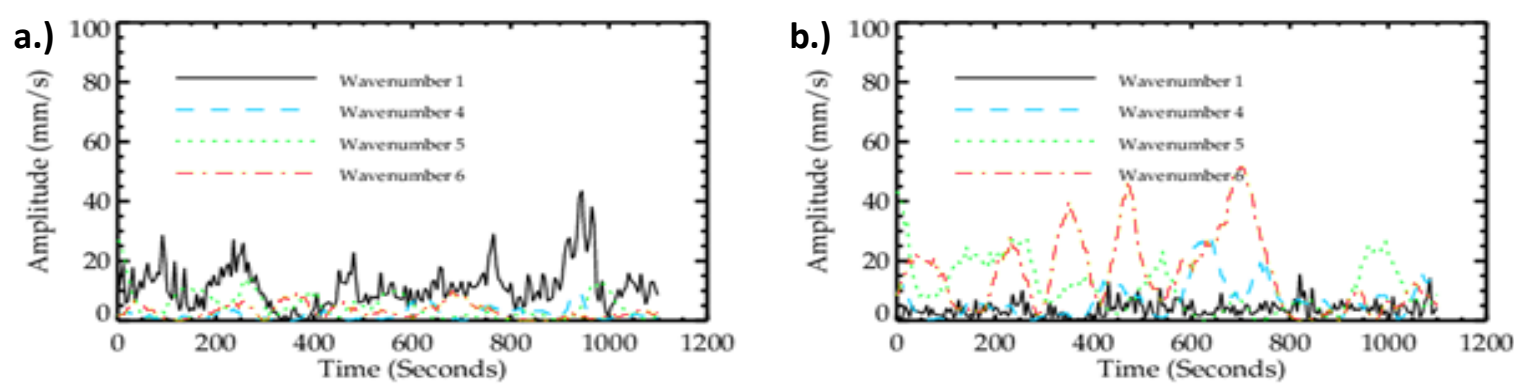

Figure 15: Velocity amplitude for selected wavenumbers for Level 3 (mid-depth) at $\Omega=1.6 \mathrm{rads}^{-1}, \Delta T=4 \mathrm{~K}$, $\mathcal{T}=1.454 \times 10^{8}, \Theta_{\mathrm{T}}=0.124 ;$ a.) azimuthal velocity, b.) radial velocity. 
From Figure 15, it can be determined from where the 'irregular' regime originates. It can be noted that the previously dominant wavenumber-3 component is no longer the largest in amplitude. The azimuthal profile is now dominated for most of the time by an irregularly-varying wavenumber- 1 , and the radial velocity profile is dominated by much higher wavenumbers, chiefly wavenumbers 4,5 and 6. These high wavenumber radial velocity amplitudes are also now much larger than the azimuthal amplitudes, which is also consistent with the irregularly-spaced small eddies and other fine-scale features found in the streakline images seen in the 'irregular' regime in Figure 10. If the Hide number is increased, these radial components can be seen to steadily increase in amplitude as well.

By examining the phases over time of the first ten wavenumbers, most of the wave components were found to be steadily drifting, with the exception of wavenumber-1. As the 'irregular' regime flow is dominated by wavenumber-4, wavenumber-5 and wavenumber- 6 components in radial velocity, Figure 16 shows the phase of these notable wavenumbers, as well as the wavenumber- 1 component, for the same flow case as in Figure 15. 

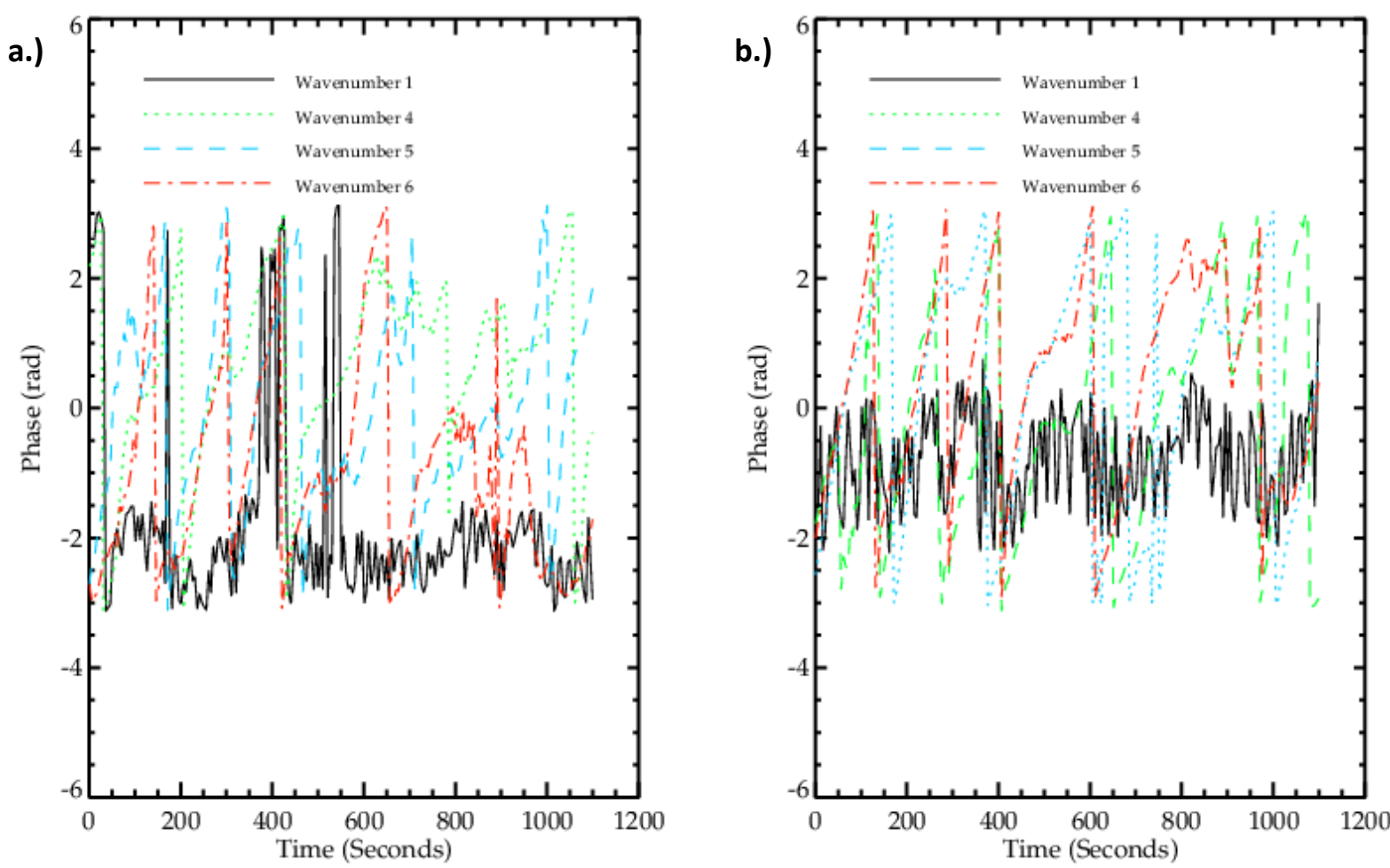

Figure 16: Wavenumber-1 phase (black) against wavenumber-4 phase (blue), wavenumber-5 phase (green) and wavenumber-6 phase (red), for Level 3 (mid-depth), $\Omega=1.6 \mathrm{rads}^{-1}, \Delta T=4 K, \mathcal{T}=1.454 \times 10^{8}, \Theta_{\mathrm{T}}=0.124$;

a.) azimuthal velocity, $b$.) radial velocity.

Figure 16 shows that the wavenumber- 1 component is quasi-stationary (the "peaks" that can be observed are merely the phase rolling over from $-\pi$ to $+\pi$ ), but the majority of the other components are still drifting. This stationary wavenumber- 1 component is likely associated with the wavenumber- 1 component of the mechanical topographic base (though this topographic configuration is not wholly wavenumber-1 in structure). Furthermore, by looking at the radial phase variations in (b), it can be noticed that wavenumber-4, wavenumber-5 and wavenumber- 6 appear to show good evidence of phase synchronisation, despite the noise of the experiment, suggesting the activity of resonant wave-triads. As discussed by Marshall and Read (2015) (initially from Bretherton 1964), the two conditions for a resonant wave-triad are that

$$
k_{m}=k_{m^{\prime}}+k_{m \prime \prime}
$$

and 


$$
\omega_{m}=\omega_{m \prime}+\omega_{m \prime \prime}
$$

where $m, m^{\prime}$, and $m^{\prime \prime}$ are the three wave modes that make up the triad, $k$ is the wavenumber of each mode, and $\omega$ is the drift frequency of each mode. Assuming that the wavenumber- 1 topography plays a role in the interactions (which is judged to be reasonable due to the dominant stationary azimuthal velocity wavenumber-1 component), there are therefore two likely triads: one involving wavenumbers 4, 5 and the stationary, topographically-anchored wavenumber- 1 component, and another involving the same stationary, topographically-anchored wavenumber-1 component and wavenumbers 5 and 6 .

Further to the above, as noted by Früh and Read (1997), integrating Equation 4 with respect to time gives the following time-independent relation:

$$
\varphi_{m}+\varphi_{m \prime}+\varphi_{m \prime \prime}=\text { const }
$$

where $\varphi$ is the phase of each mode. The relative importance of a wave-triad to the dynamic evolution of the flow can be measured by how constant the summation in Equation 5 remains over time. Hence, if the phases are 'unwrapped' then the behaviour of this phase difference over time between them can be observed, as in Figure 17. This shows the differences between the wavenumber-5, wavenumber-4 and wavenumber- 1 components and between the wavenumber- 6 , wavenumber- 5 and wavenumber- 1 components. These differences are both found by subtracting the unwrapped phase of the two middle wavenumber components (wavenumber- 4 and wavenumber-5, respectively) from the sum of the unwrapped phase of the higher wavenumber (wavenumber-5 and wavenumber-6, respectively) and wavenumber-1 components. 


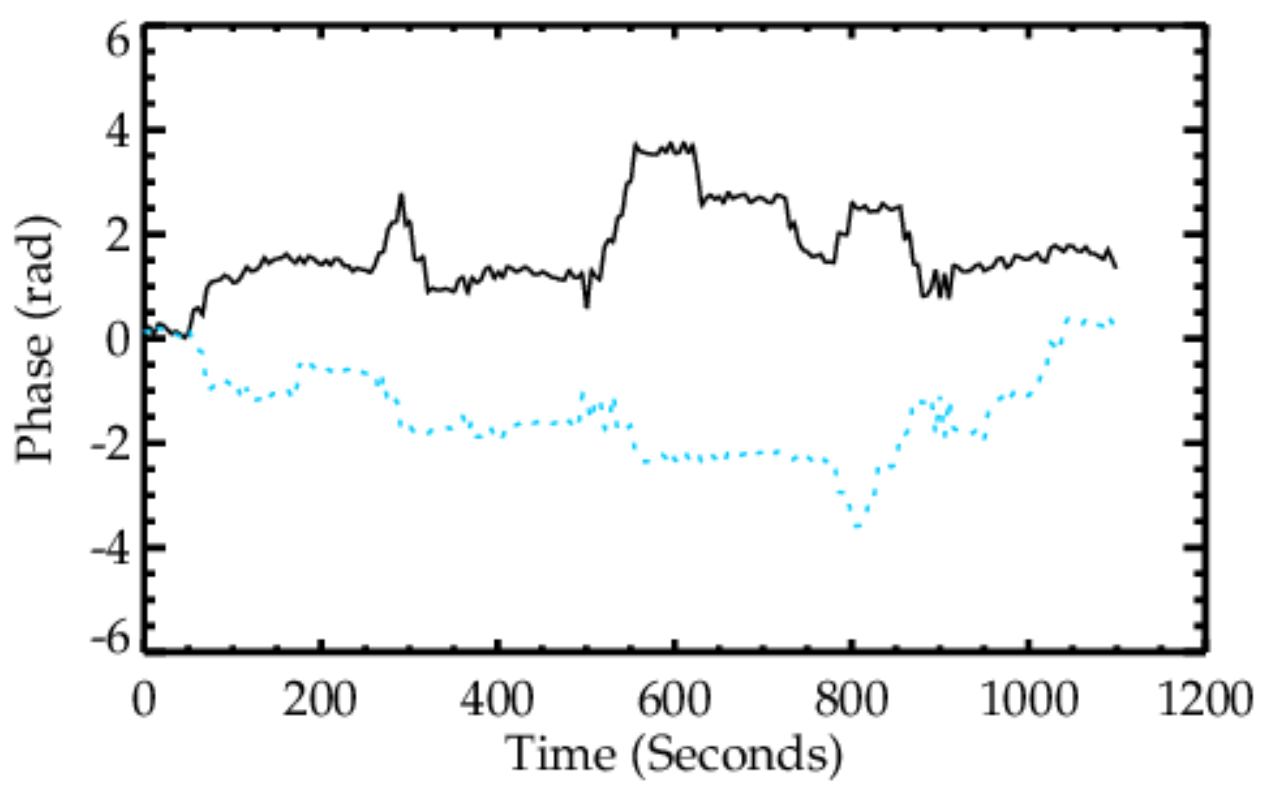

Figure 17: Unwrapped radial wavenumber-5, wavenumber-4 and wavenumber-1 phase difference $\left(\varphi_{1}+\varphi_{5}-\right.$ $\varphi_{4}$, solid black line) against unwrapped radial wavenumber-6, wavenumber-5 and wavenumber-1 phase difference $\left(\varphi_{1}+\varphi_{6}-\varphi_{5}\right.$, dotted blue line) at $\Omega=1.6 \mathrm{rads}^{-1}, \Delta T=4 K, \mathcal{T}=1.454 \times 10^{8}, \Theta_{\mathrm{T}}=0.124$.

In Figure 17, the phase difference has long intervals of near-stationarity, giving evidence that these are indeed components of significantly coherent wave-triads for the evolution of the flow. Besides the noise, there are also frequent phase-slips where the phase difference suddenly changes to a new constant value. These often occur at the same time for both lines, and with fairly regular intervals of about 150 - 200s. From Figure 4, it was found that the time taken for one cycle of the topographic interaction is roughly 180 s, so it is therefore likely that these phase-slips may be associated with interactions with the topography as the flow drifted over the peak of the base ridge.

Hence, it appears that the 'irregular' regime involves the action of two resonant wave-triads: one between the stationary wavenumber-1 component, most likely forced by the topographic base, and the drifting wavenumber- 4 and wavenumber- 5 components; and the other again between the stationary wavenumber- 1 component, but instead interacting with the drifting wavenumber- 5 and wavenumber- 6 components. In the literature, clusters of two triads that are connected by a common mode are referred to as "butterflies”. Kartashova and L'vov (2007), for example, found evidence for three such 
"butterflies” playing a role in the dynamics of their numerical investigation into LFV in the Earth's atmosphere. Kartashova and L'vov put forward the idea that intraseasonal atmospheric oscillations could be described via "periodical energy exchange” within various planetary wave-triads, including between the three "butterflies" that were able to exchange energy through their shared common modes. Once again, this gives evidence that the nonlinear interactions that cause the transition from the 'interference' regime to the 'irregular’ regime have strong wave-wave components, with energy being transferred between the different wavenumber modes. From approximately 500 - 900s of Figure 17, the phase differences are slightly more erratic than at other times, especially for the wave-triad induced by the topographic base involving the drifting wavenumber-4, wavenumber-5 and stationary wavenumber1 components. As this effect does not correspond to any notable flow phenomena occurring at the same time during this run, either visually or from wavenumber analysis, the most likely cause is a temporary increase in experimental noise. In any case, the phase differences observed in this section remain relatively small (i.e. $<\pi$ ) throughout.

In addition, for the 'irregular' regime, a contrast can be made between the time variations of the kinetic energy of the dominant wave components and the kinetic energy of the zonal mean flow. Figure 18 shows the variation over time of the kinetic energy of the zonal mean from the same points in parameter space as in Figure 15, but at the higher Level 2, and with a superposition of the total kinetic energy from the first ten wavenumbers (a proxy for the total eddy kinetic energy, since there is no clear dominant wavenumber component) for comparison. 


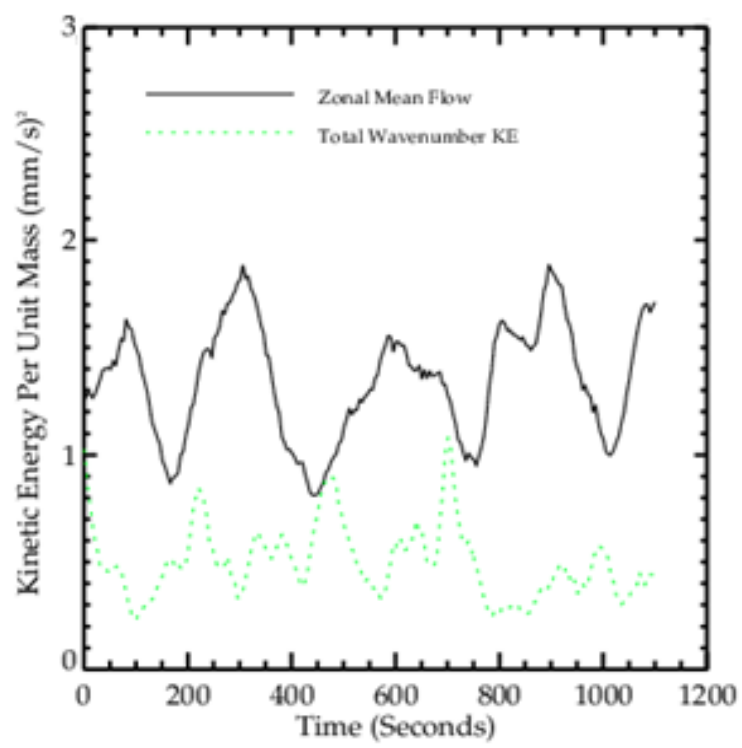

Figure 18: Radially-averaged kinetic energy of zonal mean flow (solid black line) with total kinetic energy (dotted green line, not to scale) as comparison, for Level 2 at $\Omega=1.6 \mathrm{rads}^{-1}, \Delta T=4 K, \mathcal{T}=1.454 \times 10^{8}, \Theta_{\mathrm{T}}=$ 0.124 .

Within the 'irregular' region, Figure 18 shows that there is a much less pronounced correlation between the activity of the dominant component and the mean flow. However, some weak oscillating in quadrature still seems to occur, though much less obviously than in the 'interference' regime (Figure 14). It is possible that the flow structure and resulting time-dependence of the 'irregular' regime are associated more with nonlinear wave-wave interactions, rather than with wave-zonal flow interactions, but the latter interactions are by no means absent from the dynamics of the flow. Alternatively, the irregular oscillations in the zonal mean flow might imply nonlinear interactions more complex than simple wave interference, such as the nonlinear interference vacillation mechanism as described by Ohlsen and Hart (1989). 


\section{Vertical Structure}

By observation of the vertical structure of various flow patterns obtained in these Partial Barrier experiments, the differences between the blocked and unblocked parts of the flow can be investigated. Figure 19 gives a snapshot of the vertical structure in streakline images taken close to the location in parameter space of the transition between the 'interference' and 'irregular' regimes. Each image is taken roughly 10 seconds apart, to allow for construction of a streakline picture. Considering the slow drift speed and oscillation timescales, this was determined to be sufficiently frequent. 
a.)

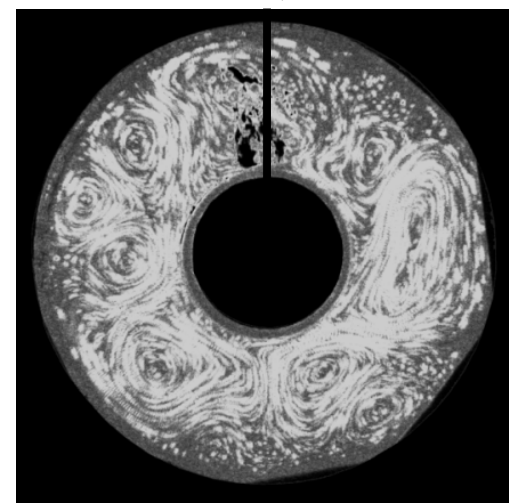

c.)

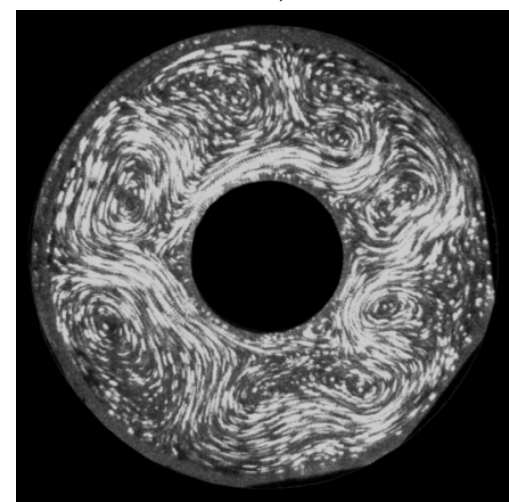

b.)

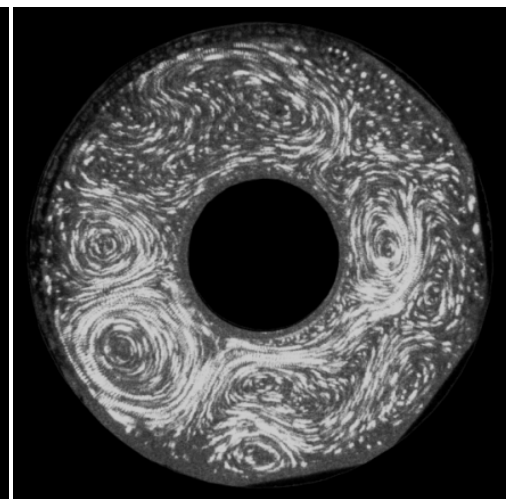

d.)

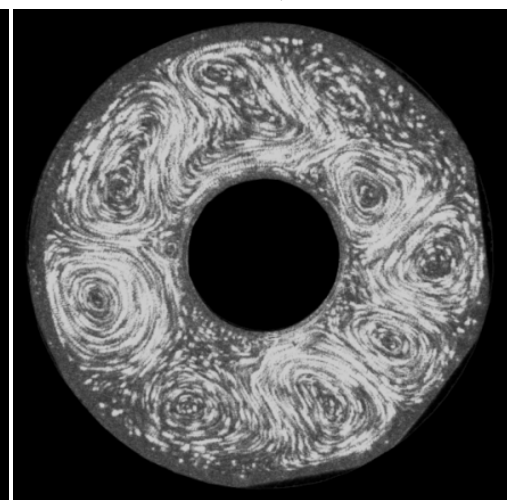

e.)

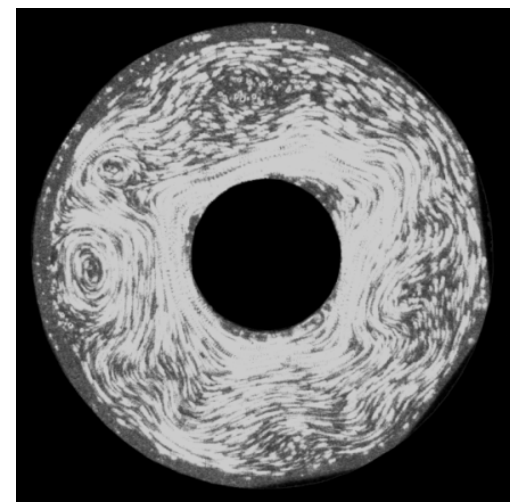

Figure 19: Sequence of almost simultaneous streakline images at each of five vertical levels at $\Omega=1.1 \mathrm{rads}^{-1}$, $\Delta T=4 K, \mathcal{T}=6.873 \times 10^{7}, \Theta_{\mathrm{T}}=0.261 ;$ a.) Level 5, b.) Level 4, c.) Level 3, d.) Level 2, e.) Level 2, f.) Level 1. Each image represents a 10s exposure to obtain the streak patterns, and is separated from the next image by 10s. A black line denotes the topographic peak in (a).

Figure 19 shows that the azimuthally-localised wave in (a) is also felt at Level 4, in (b), despite the topography not physically extending that high. The wave is visibly weaker, however, probably due to increased distance from the topography, and slightly shifted downstream (clockwise). Above that, the middle Level 3, shown at (c), exhibits a more regular wavenumber-3, as expected at this point in parameter space, with little visible evidence of the trapped wave. The components of the wavenumber- 
3 structure have yet to encounter the topography as they drift, being the equivalent of (a) or (g) in Figure 4. Higher still, (d) shows that the wavenumber-3 has been shifted further downstream, and has become more irregular. It appears the flow is transitioning towards wavenumber-4, which again is not unusual for this area of parameter space as it is so close to the 'irregular' regime and the 'transition' region in Figure 3. At the top of the annulus, (e) shows that the sloped lid appears to be suppressing baroclinic wave activity, with the flow showing only a very weak wavenumber-3 or wavenumber-4 pattern. Once again, the wave's locations can be seen to have been shifted downstream as the height is increased.

\section{Discussion}

Compared to previous studies with periodic bottom topography, such as by Li et al. (1986), Weeks et al. (1997) and the otherwise similar Marshall and Read (2015), the present Partial Barrier experiments exhibited a lack of dominant stationary wave activity throughout the whole of the investigated parameter space, but still showed evidence of topographic interactions as the wave structures drifted over the topographic peak. This interaction created flows similar to the blocked and zonal states found in those studies. Unlike with periodic bottom topography, however, the regime diagram of experiments with a partial barrier was found to be separated into two separate and distinct regions. The first was designated an 'interference' regime, found at low Taylor number, wherein a stationary wavenumber-1, mainly forced by the topography, interacted with the dominant drifting baroclinic wave and led to constructive or destructive interference which manifested itself in the form of radial jet-shifting as the waves crossed the peak of the topographic ridge.

The other main regime observed was an 'irregular' region, typically found at high Taylor number, where groups of resonant wave-triads were found to be associated with a transition toward more erratic and disordered types of flow. The isolated ridge led to the formation of a coherent "butterfly" of Rossby-like waves, made up of two nonlinear resonant wave-triads sharing a common wavenumber-1 mode. In one set of cases, one triad was between the stationary wavenumber-1 
component forced by the topography and drifting wavenumber-4 and wavenumber- 5 components; the second triad was between the same stationary wavenumber-1 component forced by the topography and drifting wavenumber-5 and wavenumber- 6 components. This would appear to indicate that the flows are dominated by nonlinear wave-wave interactions involving a stationary wave forced by the topography. The occurrence of wave-triads comprised of modes $\mathrm{M}, \mathrm{M} \pm 1$ and 1 and with a shared wavenumber-1 mode was put forward by Hide et al. (1977), suggested to arise due to nonlinear wave coupling. Furthermore, Plumb (1977) described the impact of these resonant triad interactions on the stability of Rossby waves in a long channel, noting that the wave-triad instabilities "will probably lead to a wave entirely losing its identity”, much like the transition between 'interference' and 'irregular' regimes in the current experiments. This is in contrast to the experiments of James et al. (1981), wherein no evidence of resonant wave-triad interactions was noted, and the nonlinear instability in Hide et al.'s study was argued to be associated with forcing from a thermocouple ring. As the current Partial Barrier experiments do not include a thermocouple ring or similar obstruction to the flow, yet still demonstrate dominant resonant wave-triads, Hide et al.'s proposition appears to have been validated.

Within the 'interference' region, the topography clearly has major impacts on the flow, causing jet-shifting effects as the waves cross the peak. Where the dominant wavenumber amplitude is at its weakest, the flow resembles a typical blocked structure, such as may be found in the atmosphere. When the waves are not interacting with the topography, the flow resembles the zonal state more closely. Atmospheric blocked and zonal states, however, are by their nature stationary. Egger (1978) described blocking as arising from the nonlinear interactions of slowly drifting free waves and stationary waves caused by "geographically fixed" forcing, such as at topographic ridges. Hence, the foremost wavenumber-1 component of the topographic base in this study created a non-dominant stationary wavenumber-1, which in turn interacted with the drifting dominant wavenumbers to form an oscillation between blocked and zonal states. When the stationary and drifting waves are in phase, visually denoted by one of the cyclonic eddies crossing the peak of the topography, they destructively interfere, creating a blocked flow. When they are out of phase, during the rest of the eddy's drift, they constructively interfere, creating the zonal flow state. This agrees well with the results of Harlander et al. (2012) and 
the observations of Leach (1981). The results of this study also agree with the literature in other ways, with the flows that resemble the blocked state exhibiting reduced jet intensity but larger wave amplitude, along with a noticeable increase in the strength of anticyclonic eddies when compared to those that resemble the zonal state, as noted by Weeks et al. (1997). It can be noted in this investigation that topography, the sloped lid and a reversed temperature gradient were all necessary for the 'interference' and 'irregular' regimes to appear.

\subsection{Analysis of Partial Barrier Experimental Results}

The Partial Barrier experiment was designed to block part of the working fluid, allowing comparison between blocked flow at the lower levels and unblocked flow at the higher levels. The blocked bottom flow was found to be defined by a large persistent azimuthally-trapped wave upstream of the topographic peak, as well as smaller, irregularly-spaced downstream eddies. This is quite different to the results of Wordsworth's (2009) fully blocked ocean basin experiments, which found a quasi-barotropic jet rather than trapped waves. It is likely, therefore, that in a Partial Barrier experiment, the existence of the unblocked flow affects the structure of the blocked flow. Similarly, the azimuthallylocalised wave extends further vertically than the topography, weakening as it crosses into the unblocked flow. This bears some similarity to real oceanic flow, as reviewed e.g. by Huthnance (1989) and Martínez and Allen (2004). The lower levels are found to be dominated by a stationary wavenumber-1 component, presumably associated with a topographically trapped wave, which is also found at a reduced amplitude at all other heights. Further into the unblocked flow, the circulation is far more regular, with structure depending on the location in parameter space, and with little visible evidence of the azimuthally-localised wave, due to its weakened state. Above this point, the flow is reasonably uniform in the vertical direction, appearing mostly the same in structure at all heights, except for a notable reduction in wave amplitude near the sloped lid. As such, the major difference between the blocked and unblocked flows was found to be the notable azimuthally-localised, stationary wave in 
the former.

Whilst the base used in these experiments consisted of a spread of zonal wavenumbers, not only of wavenumber-1 in azimuthal structure, it would still be reasonable to conclude that resonant interactions would likely occur mainly between the topography and a wavenumber- 1 flow. However, despite a topographically-forced stationary wavenumber-1 component being noted, especially at high Taylor number, this component was never found to dominate the flow structure, and thus, in contrast to earlier work (e.g. Read and Risch 2011; Risch and Read 2015; Marshall and Read 2015), no clear evidence of topographic resonance was discovered.

\subsection{Oceanic Blocking}

In the blocked sections of the ocean basin, Huthnance (1989) and Martínez and Allen (2004) note that trapped waves are a common and dynamically important aspect of the flow, with an impact on the ocean circulation on the same order as that of the wind forcing, according to the latter. The blocked bottom flow in the Partial Barriers study was found to be defined by a large, persistent, azimuthally-localised wave upstream of the topographic peak, as well as smaller, irregularly-spaced downstream eddies. The azimuthally-trapped wave also extended further vertically than the topography, causing a stationary wavenumber-1 component to appear in the flow structures at higher levels, but weakened in magnitude as it crossed into the unblocked flow. This again bears some similarity to the real oceanic flow found near coastal boundaries. On the other hand, these results are quite different to those of Wordsworth's (2009) fully blocked ocean basin experiments, which found a quasi-barotropic jet rather than trapped waves. This quasi-barotropic jet was suggested to be an equivalent to the western boundary currents of oceanic flows, similar to the observations discussed by Rayer et al. (1998). It is likely, therefore, that in the Partial Barrier experiment the existence of the unblocked flow affected the structure of the blocked flow, as fluid could move vertically over the curved surface of the isolated ridge instead of generating a trapped jet structure. In this way, further experiments replacing the curved ridge 
with a sheer barrier would be needed to investigate whether trapped jets, and thus evidence for western boundary currents in the annulus, can occur under partial barrier conditions.

\subsection{Multiple Equilibria}

Throughout all the conducted experiments here, as well as in the previous studies with periodic topographic of Marshall and Read (2015), only limited evidence of multiple equilibria in the annulus was observed, consistent with what was argued by Tung and Rosenthal (1985) and Cehelsky and Tung (1987). Locations of hysteresis or alternative states at the same point in parameter space were found to occur rarely, if at all. Due to this, the flow was therefore unable to 'choose' between these distinct states, based on initial conditions. On the other hand, some evidence was found for the occurrence of the blocked and zonal states themselves. Within the 'interference' regime the flows that visually resembled the blocked state exhibited reduced jet intensity but larger wave amplitude, along with a noticeable increase in the strength of anticyclonic eddies when compared to those that resembled the zonal state. This is exactly how the two states are defined in the Earth's atmosphere, as noted by Weeks et al. (1997), suggesting that the zonal and blocked states themselves come about from interactions between drifting waves and a stationary wave. Evidence of this stationary wave is present at all depths and all sections of parameter space, and was found to dominate at the bottom of the annulus, regardless of whether the flow was physically blocked or not. However, in both cases, the two states were part of a regular oscillation with periodic transitions between them, and lacked the permanence required for multiple equilibria, unlike what was previously noted by Risch (1999). 


\subsection{Atmospheric Flow Structure}

In Marshall and Read (2015) it was discussed that dominant stationary waves in the atmosphere are commonly found to have westward phase shifts with height (see Reinhold and Pierrehumbert 1982 and James 1994), showing a strong poleward momentum and temperature flux, as well as vertically propagating waves (see Holton 1992 and Pedlosky 1987). Despite the flow in the present experiments being found to drift constantly throughout all of parameter space, interacting with the topography only via interference effects at high Hide numbers (the 'interference' regime) and leading to erratic, multiwavenumber flows at low Hide numbers (the 'irregular’ regime), a phase shift with height was found in the vertical structure of the fluid throughout the whole of the examined parameter space. These phase shifts with height were also always found to be tilting downstream, similar to that shown by laboratory experiments with periodic topographic bases (see Leach 1981 and Marshall and Read 2015), though the opposite of the upstream tilt noted in atmospheric observations (likely due to the reversed temperature gradient of the annulus' walls). As suggested in Marshall and Read, to investigate the associated poleward momentum and temperature flux further, in addition to vertically propagating waves, it would be useful to conduct experiments in a numerical model of the annulus, taking measurements of the above properties throughout the entire fluid.

The 'irregular' region of the present experiments was associated with the occurrence of linked resonant wave-triads in the form of a "butterfly". This suggested a possible connection to LFV, as Kartashova and L’vov (2007) found three such "butterflies” in their numerical investigation into LFV. In addition, Marshall and Read's (2015) experiments with periodic topography also found notable resonant wave-triads with oscillation periods on the timescale of intraseasonal atmospheric oscillations, though with lower wavenumber components.

On the subject of atmospheric climate change, Petoukhov et al. (2012) suggested that severe weather events are due to a rise in number and longevity of persistent blocked events and stationary waves, with a root cause of atmospheric resonant wave-triads made of high wavenumbers 
(wavenumber-6, wavenumber-7 and wavenumber-8 in that study). The present experiments do indeed demonstrate the occurrence of high wavenumber resonant wave-triads, but this was associated more with the occurrence of the erratic 'irregular' regime, rather than persistent blocked events. Furthermore, as discussed previously, no evidence was found of distinct, alternative blocked and zonal states, and both dominant stationary waves and topographic resonance were only observed with fully periodic topography (see Marshall and Read 2015).

\section{References}

Berggren, R., Bolin, B. \& Rossby, C-G., 1949. An Aerological Study of Zonal Motion, its Perturbations and Break-down. Tellus, 1(2), 14-37.

Bretherton, F.P., 1964. Resonant Interactions between Waves. The Case of Discrete Oscillations. J. Fluid Mech., 20(3), 457-479

Cehelsky, P. \& Tung, K.K., 1987. Theories of Multiple Equilibria and Weather Regimes - A Critical Reexamination. Part II: Baroclinic Two-Layer Models. J. Atmos. Sci., 44(21), 3282-3303.

Charney, J.G. \& DeVore, J.G., 1979. Multiple Flow Equilibria in the Atmosphere and Blocking. J. Atmos. Sci., 36(7), 1205-1216.

Charney, J.G. \& Drazin, P.G., 1961. Propagation of Planetary-Scale Disturbances from the Lower into the Upper Atmosphere. J. Geophys. Res., 66(1), 83-109.

Charney, J.G. \& Straus, D.M., 1980. Form-Drag Instability, Multiple Equilibria and Propagating Planetary Waves in Baroclinic, Orographically Forced, Planetary Wave Systems. J. Atmos. Sci., 37(6), 1157-1176.

Egger, J., 1978. Dynamics of Blocking Highs. J. Atmos. Sci., 35(10), 1788-1801. 
Fincham, A.M. \& Spedding, G.R., 1997. Low Cost, High Resolution DPIV for Measurement of Turbulent Fluid Flow. Experiments in Fluids, 23(6), 449-462.

Fowlis, W.W. \& Hide, R., 1965. Thermal Convection in a Rotating Annulus of Liquid: Effect of Viscosity on the Transition between Axisymmetric and Non-Axisymmetric Flow Regimes. J. Atmos. Sci., 22, 541-558.

Früh, W-G. \& Read, P.L., 1997. Wave Interactions and the Transition to Chaos of Baroclinic Waves in a Thermally Driven Rotating Annulus. Phil. Trans. Math. Phys. Eng. Sci., 355(1722), 101-153.

Harlander, U., Wenzel, J., Alexandrov, K., Wang, Y. \& Egbers, C., 2012. Simultaneous PIV and thermography measurements of partially blocked flow in a differentially heated rotating annulus, Exp. Fluids, 52(4), 1077-1087

Hide, R., 1958. An Experimental Study of Thermal Convection in a Rotating Liquid. Phil. Trans. $R$. Soc.Lon. A, 250(983), 441-478.

Hide, R. \& Mason, P.J., 1975. Sloping Convection in a Rotating Fluid. Advances in Physics, 24(1), $47-100$.

Hide, R., Mason, P.J. \& Plumb, R.A., 1977. Thermal Convection in a Rotating Fluid Subject to a Horizontal Temperature Gradient: Spatial and Temporal Characteristics of Fully Developed Baroclinic Waves. J. Atmos. Sci., 34(6), 930-950.

Holton, J.R., 1992. An Introduction to Dynamic Meteorology. $3^{\text {rd }}$ ed. New York: Academic Press. Huthnance, J.M., 1989. Internal Tides and Waves near the Continental Shelf Edge. Geophys. Astrophys. Fluid Dyn., 48(1-3), 81-106.

James, I.N., 1994. Introduction to Circulating Atmospheres, Cambridge: Cambridge University Press. James, I.N., Jonas, P.R. \& Farnell, L., 1981. A Combined Laboratory and Numerical Study of Fully Developed Steady Baroclinic Waves in a Cylindrical Annulus. Q. J. R. Meteorol. Soc., 107(451), 51- 
78.

Jin, F.F. \& Ghil, M., 1990. Intraseasonal Oscillations in the Extratropics: Hopf Bifurcations and Topographic Instabilities. J. Atmos. Sci., 47(24), 3007-3022.

Kartashova, E. \& L’vov, V.S., 2007. Model of Intraseasonal Oscillations in Earth’s Atmosphere. Phys. Rev. Lett. 98(19), 198501(4).

Leach, H., 1981. Thermal Convection in a Rotating Fluid: Effects due to Bottom Topography. J. Fluid Mech., 109, 75-87.

Li, Q.G., Kung, R. \& Pfeffer, R.L., 1986. An Experimental Study of Baroclinic Flows with and without Two-Wave Bottom Topography. J. Atmos. Sci., 43(22), 2585-2599.

Marshall, S.D. \& Read, P.L., 2015. An Experimental Investigation into Topographic Resonance in a Baroclinic Rotating Annulus. Geophys. Astrophys. Fluid Dyn., 109(4), 391-421.

Martínez, J.A. \& Allen, J.S., 2004. A Modeling Study of Coastal-Trapped Wave Propagation in the Gulf of California. Part I: Response to Remote Forcing. J. Phys. Oceanogr., 34(6), 1313-1331.

Mason, P.J., 1975. Baroclinic Waves in a Container with Sloping End Walls. Phil. Trans. R. Soc. Lond. A, 278(1284), 397-445.

Ohlsen, D.R. \& Hart, J.E., 1989. Nonlinear Interference Vacillation. Geophys. Astrophys. Fluid Dyn., 45(3-4), 213-235.

Pedlosky, J., 1987. Geophysical Fluid Dynamics. $2^{\text {nd }}$ ed. New York and Berlin: Springer-Verlag.

Petoukhov, V., Rahmstorf, S., Petri, S. \& Schellnhuber, H.J., 2013. Quasiresonant Amplification of Planetary Waves and Recent Northern Hemisphere Weather Extremes. Proceedings of the National Academy of Sciences, 110(14), 5336-5341.

Plumb, R.A., 1977. The Stability of Small Amplitude Rossby Waves in a Channel. J. Fluid 
Mech., 80(4), 705-720.

Rayer, Q.G., Johnson, D.W. \& Hide, R., 1998. Thermal Convection in a Rotating Fluid Annulus Blocked by a Radial Barrier. Geophys. Astrophys. Fluid Dyn., 87(3), 215-252.

Read, P.L., Bel, M.J., Johnson, D.W., \& Small, R.M. 1992. Quasi-periodic and chaotic flow regimes in a thermally driven, rotating fluid annulus. Journal of Fluid Mechanics, 238, 599-632.

Read, P.L. \& Risch, S.H., 2011. A Laboratory Study of Global-Scale Wave Interactions in Baroclinic Flow with Topography, Part I: Multiple Flow Regimes. Geophys. Astrophys. Fluid Dyn., 105(2-3), 128-160.

Reinhold, B.B. \& Pierrehumbert, R.T., 1982. Dynamics of Weather Regimes: Quasi-Stationary Waves and Blocking. Mon. Wea. Rev., 110(9), 1105-1145.

Risch, S.H., 1999. Large-Scale Wave Interactions in Baroclinic Flow with Topography. DPhil. University of Oxford.

Risch, S.H. \& Read, P.L., 2015. A Laboratory Study of Global-Scale Wave Interactions in Baroclinic Flow with Topography, Part II: Vacillations and Low-Frequency Variability. Geophys. Astrophys. Fluid Dyn., 109(4), 359-390.

Taylor, G.I. (1923). Stability of a Viscous Liquid Contained Between Two Rotating Cylinders. Phil. Trans. R. Soc. Lon. A, 223(612), 289-343.

Tian, Y., Weeks, E.R., Ide, K., Urbach, J.S., Baroud, C.N., Ghil, M. \& Swinney, H.L., 2001. Experimental and Numerical Studies of an Eastward Jet over Topography. J. Fluid Mech., 438, 129157.

Tung, K.K. \& Rosenthal, A.J., 1985. Theories of Multiple Equilibria and Weather Regimes - A Critical Reexamination. Part I: Barotropic Models. J. Atmos. Sci., 42(24), 2804-2819.

Weeks, E.R., Tian, Y., Urbach, J.S., Ide, K., Swinney, H.L. \& Ghil, M., 1997. Transitions Between 
Blocked And Zonal Flows In A Rotating Annulus With Topography. Science, 278(5343), 1598-1601

Woollings, T., 2010. Dynamical Influences on European Climate: An Uncertain Future. Phil. Trans.

R. Soc. Lon. A, 368(1924), 3733-3756.

Wordsworth, R.D., 2009. Theoretical and Experimental Investigations of Turbulent Jet Formation in Planetary Fluid Dynamics. DPhil. University of Oxford.

Wordsworth, R.D., Read, P.L. \& Yamazaki, Y.H., 2008. Turbulence, Waves, and Jets in a Differentially Heated Rotating Annulus Experiment. Phys. Fluids, 20(12), 126602. 